\title{
As aves da Estação Ecológica Serra das Araras, Mato Grosso, Brasil
}

\author{
Rafael Martins Valadão ${ }^{1,2}$ \\ ${ }^{1}$ Instituto Chico Mendes de Conservação da Biodiversidade, ESEC Serra das Araras, Rod. MT 343, \\ Km 69, Comunidade Salobra Grande, Zona Rural, CEP 78398-000, Porto Estrela, MT, Brasil \\ ${ }^{2}$ Autor para correspondência: Rafael Martins Valadão, e-mail: rafael.valadao@icmbio.gov.br
}

VALADÃO, R.M. Birds of the Estação Ecológica Serra das Araras, Mato Grosso, Brazil. Biota Neotrop. 12(3): http://www.biotaneotropica.org.br/v12n3/en/abstract?article+bn02612032012

\begin{abstract}
There are 1801 bird species in Brazil and 856 of those occur in Cerrado. This Biome is the largest, most distinctive and the richest Savanna in the world and it is probably the world's most endangered tropical Savanna, becoming a Hotspot and priority area for biodiversity conservation. Ecological Station Serra das Araras (EESA) occupies an area of 28.700 hectares of Cerrado, located in the Southwest of the State of Mato Grosso. It is part of the Provincia Serrana, a $400 \mathrm{~km}$ long and $40 \mathrm{~km}$ large mountain corridor connecting the Amazonian and Pantanal Biomes. The bird community of EESA was sampled monthly from May 2006 through December 2007. We placed a $500 \mathrm{~m}$ transect in each of five vegetation types. Each transect was walked once a month for 4 hours starting at sunrise and for 2 hours starting at sunset. Total sampling effort was 600 hours, comprising 80 daylight hours and 40 evening hours per transect/habitat. The list of birds of ESSA has 431 bird species, including 13 endemic birds of Cerrado and 7 threatened species in Brazil. Of these, 396 were recorded during this sampling effort, 29 out the sampling effort and six species were not registered in this study, although listed in studies conducted at EESA between 1986 and 1988. In the semi deciduous forest were recorded 150 species of birds, 256 species in riparian forest, 218 species in cerradão, 206 in cerrado sensu strict and 176 in parquet cerrado. Data analysis splitted the phytophysiognomies in two groups, the savanna (cerrado sensu strict and parque cerrado) and forested (gallery forest, cerradão and semi deciduous forest) bird communities. EESA bird richness is high compared with other areas of Cerrado. Perhaps the environmental heterogeneity of this protected area, with varied topography and vegetation is the reason for this larger species number. Along with the number of bird species, the presence of endemic and endangered species reinforces the importance of EESA for the conservation of this group.
\end{abstract}

Keywords: Cerrado, protected area, habitat use, community ecology, endemic species, red list.

VALADÃO, R.M. As aves da Estação Ecológica Serra das Araras, Mato Grosso, Brasil. Biota Neotrop. 12(3): http://www.biotaneotropica.org.br/v12n3/pt/abstract?article+bn02612032012

Resumo: O Brasil possui 1.801 espécies de aves e o Cerrado abriga 856 dessas espécies. Esse bioma que cobre $24 \%$ do território nacional é a maior, mais distinta, mais rica e, provavelmente mais ameaçada savana tropical do mundo, o que lhe tornou "hot spot" mundial e área prioritária para conservação da biodiversidade. A Estação Ecológica Serra das Araras (EESA) ocupa 28.700 hectares de Cerrado no sudoeste do Mato Grosso, faz parte da Província Serrana, um corredor de serras com cerca de $400 \mathrm{~km}$ de comprimento por $40 \mathrm{~km}$ de largura, o qual conecta o Cerrado à transição do bioma Amazônico e ao Pantanal. Com o objetivo de inventariar a comunidade de aves da EESA, foi percorrido um transecto de $500 \mathrm{~m}$ em cada uma das seguintes fitofisionomias: mata semidecídua, mata ciliar, cerradão, cerrado sentido restrito e parque cerrado. Cada transecto foi amostrado uma vez por mês, de maio de 2006 a dezembro de 2007, durantes quatro horas a partir do nascer e duas horas a partir do por do sol, totalizando 600 horas de amostragem, sendo 80 horas de dia e 40 horas de noite por transecto/hábitat. A lista de aves da ESSA possui 431 espécies de aves, incluindo treze endêmicas do Cerrado e sete nacionalmente ameaçadas de extinção. Dessas, 396 foram registradas durante o esforço amostral, 29 fora do período amostral e seis constam somente na lista de 305 espécies registradas entre 1986 e 1988. Na mata semidecídua foram registradas 150 espécies, na mata ciliar 256, no cerradão 218, no cerrado sentido restrito 206 e no parque cerrado 176 espécies de aves. Quanto à similaridade, formaram-se dois grupos, o das fitofisionomias savânicas (cerrado sentido restrito e parque cerrado) e outro das florestais (mata ciliar, cerradão e mata semidecídua). A riqueza de espécies de aves na EESA é alta quando comparada com outros estudos realizados no Cerrado e provavelmente está relacionada heterogeneidade ambiental presente nessa unidade de conservação, com relevo e vegetação variados. Além de preservar um elevado número, a presença de espécies de aves endêmicas e ameaçadas de extinção reafirmam a importância da EESA para conservação desse grupo.

Palavras-chave: Cerrado, unidade de conservação, uso de hábitat, ecologia de comunidade, espécies endêmicas, espécies ameaçadas de extinção. 


\section{Introdução}

Aves são importantes na avaliação da qualidade ambiental e peças chaves na determinação de áreas para a conservação, pois se trata de um grupo diversificado que ocupa diferentes hábitats, níveis tróficos e são altamente sensível às modificações ambientais. O comportamento conspícuo e facilidade de identificação de grande parte das espécies, além da rapidez na amostragem fazem delas um dos grupos mais bem conhecido da região neotropical (Kattan et al. 1994, Stotz et al. 1996, Tubelis \& Cavalcanti 2000, Marini 2001, Eken et al. 2004).

O Brasil é o terceiro país em riqueza de aves, com 1.801 espécies (Comitê... 2011), dessas 856 tem registros para o Cerrado e 30 são endêmicas (Silva \& Santos 2005). Esse pequeno número de espécies endêmicas pode ser explicado por sua interconexão como os demais biomas, o que influenciou no processo de especiação desse grupo (Sick 1966). Embora ocupe o terceiro lugar em riqueza de aves no Brasil, o Cerrado ainda é um ambiente pouco estudado em relação a sua avifauna, extensas porções de seu território ainda não tinham sido "minimamente estudadas" (localidades que tivessem pelo menos 80 espécimes coletados ou uma lista com no mínimo 100 espécies) em 1995, fato que pouco mudou em dez anos (Silva 1995, Silva \& Santos 2005).

O Cerrado cobre aproximadamente $24 \%$ do território nacional e é hoje a maior, mais distinta, mais rica e, provavelmente, a mais ameaçada savana tropical do mundo (Silva \& Bates 2002, Silva \& Santos 2005, Serviço... 2011), o que lhe garante o título de "hot spots" mundial e área prioritária para conservação da biodiversidade (Myers et al. 2000). Um dos principais fatores determinantes da sua riqueza é a sua estratificação horizontal, pois Cerrado pode ser entendido como um grande mosaico, no qual as peças são as diferentes fitofisionomias, as quais produzem um gradiente em densidade e altura, definido por formações campestres a florestais (Coutinho 1978).

Nos últimos anos, tem havido uma intensa substituição das áreas de vegetação nativa do Cerrado por zonas urbanas, agricultura, pastagens e reflorestamentos com espécies vegetais exóticas (Machado \& Lamas 1996). Dados do Serviço Florestal Brasileiro (2011) mostram que ao final de 2008 o percentual de área desmatada era de $47,8 \%$, e que apenas $6,4 \%$ do bioma encontrava-se formalmente preservado em unidades de conservação, valor é bem inferior aos $10 \%$ assumido, via termo de compromisso, pelo Ministério do Meio Ambiente durante o Workshop “Ações Prioritárias para a conservação da biodiversidade do Cerrado e Pantanal” (Brasil 1999).

Inventariar um local é a forma mais direta para se acessar parte dos componentes da diversidade animal de uma localidade (Silveira et al. 2010). As listas de espécies, principal produto desses inventários, são importantes para análise futura de relações biogeográficas (Ribon et al. 1995, Nunes \& Pacheco 2004), para a determinação de rotas migratórias (Ribon et al. 1995) e uma contribuição fundamental aos órgãos gestores das áreas protegidas brasileiras (Argel-de-Oliveira 1993). Podem ser utilizadas para investigação da dinâmica natural da flora e fauna, dos eventos ecológicos e da eficácia de gestão de uma Unidade de Conservação.

O objetivo deste trabalho foi inventariar a avifauna da Estação Ecológica Serra das Araras, comparar a riqueza de aves entre as fitofisionomias estudadas, comparar os resultados obtidos a estudos anteriores, bem como criar uma base de dados confiável que embase as tomadas de decisão e gestão dessa área protegida.

\section{Material e Métodos}

\section{1. Área de estudo}

A Estação Ecológica Serra das Araras é uma unidade de conservação federal que ocupa 28.700 ha do bioma Cerrado no sudoeste do Mato Grosso, nas cidades de Porto Estrela e Cáceres. Sua área está localizada entre as latitudes $15^{\circ} 27^{\prime} 11^{\prime \prime}-15^{\circ} 51^{\prime} 04^{\prime \prime} \mathrm{Sul}$ e longitudes $57^{\circ} 06^{\prime} 06^{\prime \prime}-57^{\circ} 16^{\prime} 41^{\prime \prime}$ Oeste (Figura 1).

A região da unidade de conservação destaca-se das demais áreas do entorno por se encontrar fisicamente isolada e apresentar relevo e altitudes bem diferenciados das regiões adjacentes (vale do alto rio Paragai e Pantanal), sendo parte da Província Serrana. Essa unidade geomorfológica é um extenso corredor de serras paralelas, com cerca de $400 \mathrm{~km}$ de comprimento por $40 \mathrm{~km}$ de largura, estendendo-se do Pantanal de Cáceres até a cidade de Paranatinga. O Clima da região, segundo classificação de Köppen, é do tipo Aw Megatérmico com nítida sazonalidade, sendo o período chuvoso de outubro a abril e o período seco de maio a setembro (Ross 1991).

Cinco fitofisionomias foram amostradas sistematicamente durante o presente estudo. Sua classificação obedece Ribeiro \& Walter (2008) e possuem as seguintes características:

1.1 Mata semidecídua: fitofisionomia florestal com árvores eretas, de 15 a $30 \mathrm{~m}$ de altura, que crescem sobre solo de origem calcária com afloramento rochoso. No período chuvoso a cobertura foliar é superior a $90 \%$, e diminui para menos de $50 \%$ no período seco, com a queda das folhas das espécies decíduas.

1.2 Mata ciliar: fitofisionomia florestal que apresenta árvores eretas de 20 a $25 \mathrm{~m}$ de altura. A caducifólia, durante o período seco, permite o desenvolvimento de um denso sub-bosque e garante um estoque perene de serrapilheira.

1.3 Cerradão: fitofisionomia florestal com plantas de folhas duras e coriáceas, composto por espécies vegetais tanto do cerrado sentido restrito como de mata ciliar. A altura das árvores varia de 8 a $15 \mathrm{~m}$ de altura, proporcionando condições de luminosidade que favorece a formação de estrato arbustivo.

1.4 Cerrado sentido restrito: fitofisionomia savânica com denso estrato graminoso e presença de árvores baixas, inclinadas, tortuosas, com ramificações irregulares e retorcidas que variam entre 3 e $5 \mathrm{~m}$ de altura.

1.5 Parque cerrado: fitofisionomia savânica que apresenta árvores de cerrado sentido restrito de 3 a $6 \mathrm{~m}$ de altura, agrupadas em pequenas elevações bem drenadas do terreno, os "murundus". Neles a cobertura arbórea atinge de 50 a $60 \%$ e nas depressões, cobertas por um tapete graminoso denso que cresce em solo alagado de janeiro a abril, a cobertura arbórea cai para praticamente $0 \%$.

\section{Métodos}

O levantamento da avifauna foi realizado no período de maio de 2006 a dezembro de 2007, em campanhas mensais, com intervalos médios de $27( \pm 3)$ dias entre elas.

Cinco fitofisionomias foram amostradas de forma sistemáticas: mata ciliar (Figura 2a), mata semi decídua (Figura 2b), cerradão (Figura 2c), cerrado sentido restrito (Figura 2d) e parque cerrado (Figura 2e). Um transecto de $500 \mathrm{~m}$ foi delimitado em trilhas preexistentes em cada uma das fitofisionomias (Figura 1b). Cada transecto foi amostrado uma vez por mês, percorrendo-se livremente sua extensão, durante quatro horas a partir do nascer e duas horas a partir do por do sol, totalizando 600 horas de amostragem, sendo 80 horas durante o dia e 40 horas durante a noite por transecto/hábitat.

Áreas de mata calcária (Figura 2f), campo rupestre (Figura 2g) e cerrado rupestre (Figura $2 \mathrm{~h}$ ) foram amostradas esporadicamente devido a sua dificuldade de acesso. Capturas eventuais, utilizando-se redes de neblina, foram também realizadas para confirmação de algumas espécies. Por não terem obedecido a uma metodologia padronizada, os registros obtidos nas áreas amostradas esporadicamente e nas capturas, somente serviram para compor a lista final de espécies, como registros não sistemáticos; o esforço amostral não foi considerado 


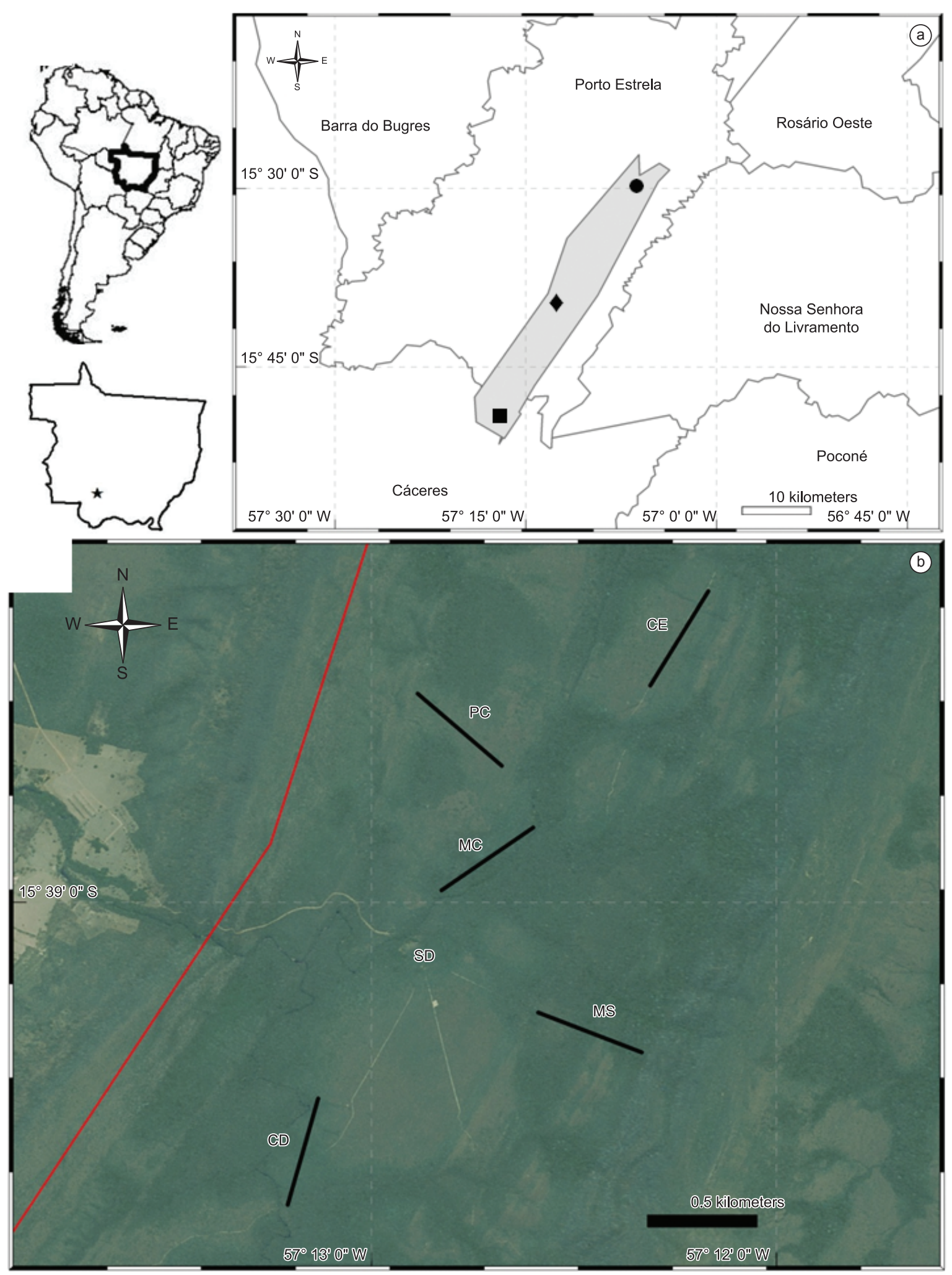

Figura 1. a) Localização da Estação Ecológica Serra das Araras (Porto Estrela e Cáceres, Mato Grosso), com indicação das áreas de amostragem da avifauna: - (pontos de amostragem sistemática); • (ponto de amostragem não sistemática: mata calcária) e a (pontos de amostragens não sistemáticas: campo e cerrado rupestres). b) Localização dos transectos nas áreas de amostragem sistemática: MS (mata semidecídua), MC (mata ciliar), CD (cerradão), CE (cerrado sentido restrito) e PC (parque cerrado). SD é a localização da sede da EESA e a linha vermelha indica o seu limite.

Figure 1. a) Location of the Serra das Araras Ecological Station (Porto Estrela e Cáceres, Mato Grosso), indicating the sampling areas: (systematic sampling spots), • (sporadic sampling spot: limestone forest) and (sporadic sampling points: campo rupestre and cerrado rupestre). b) Location of transects in the areas of systematic sampling: MS (semi-deciduos forest), MC (riparian forest), CD (cerradão) CE (cerrado sentido restrito) and PC (parque cerrado). SD is the location of EESA's headquarters and the red line is its limit. 

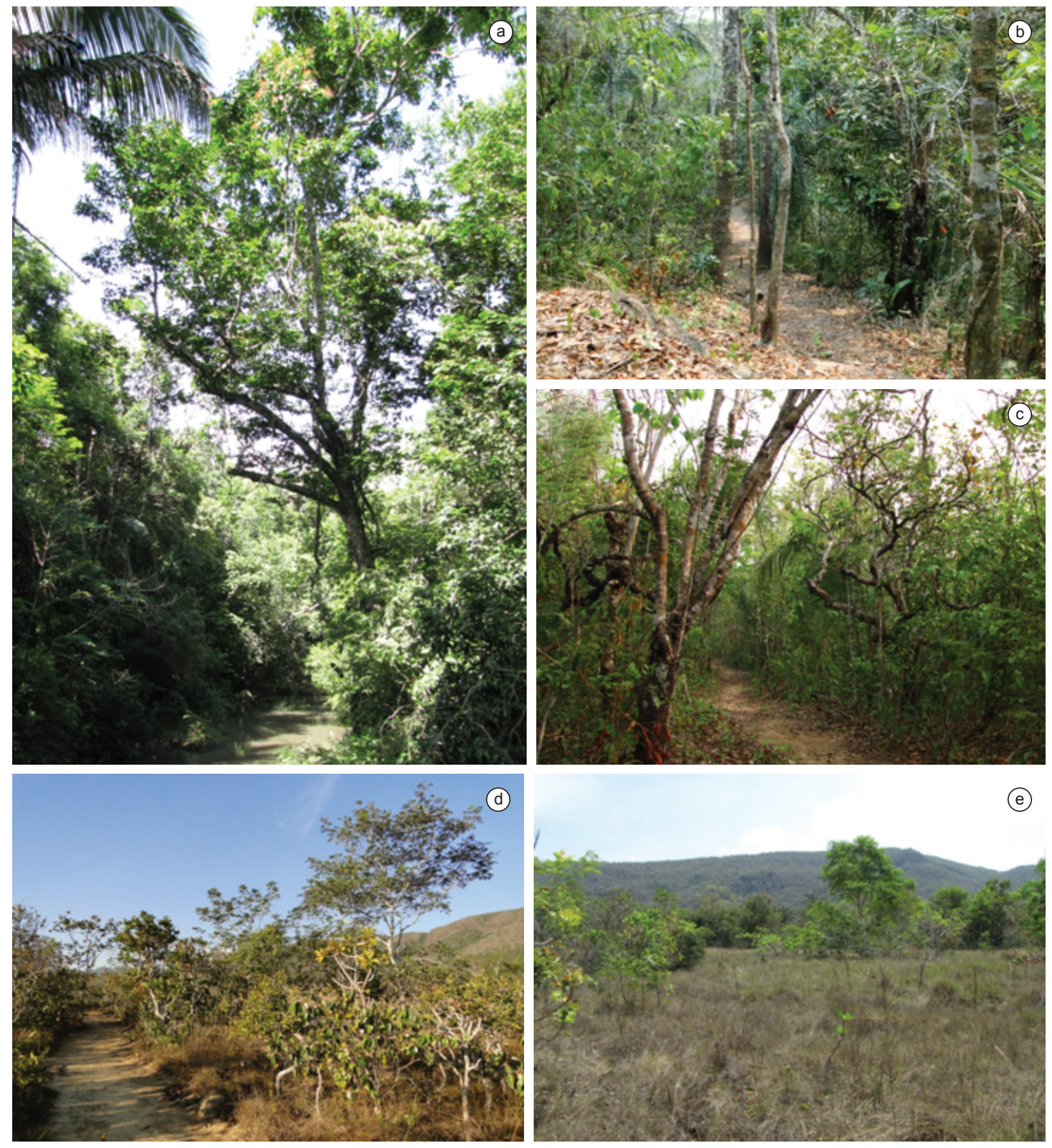

(d)

(e)
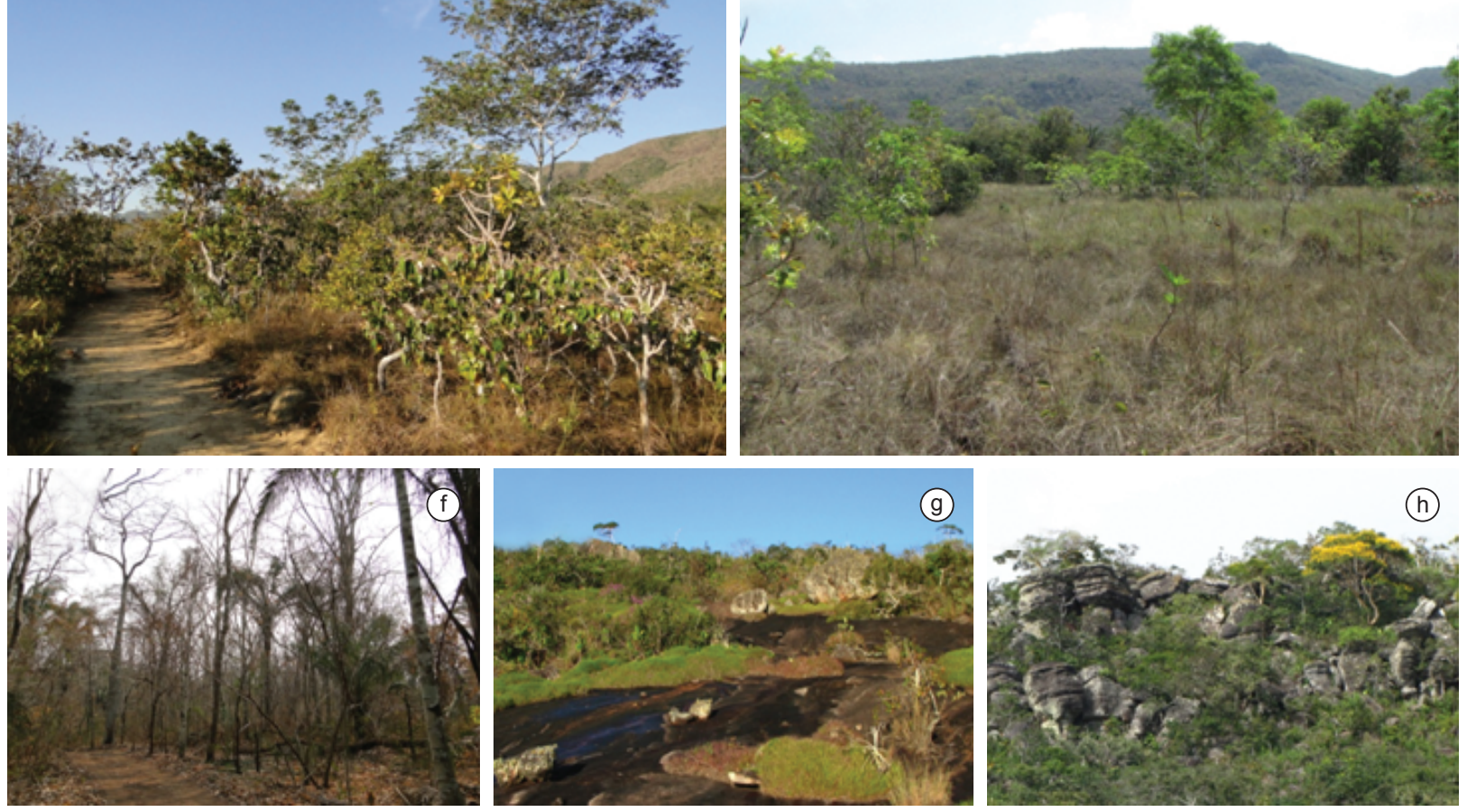

(h)

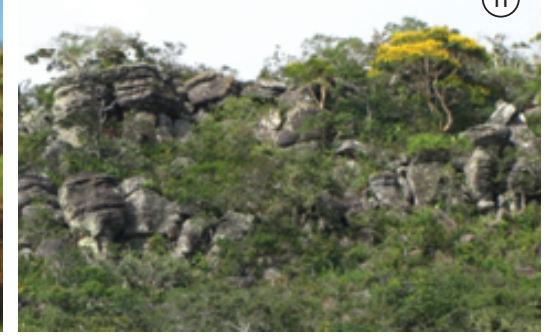

Figura 2. Fotos das áreas de estudo amostradas de forma sistemática: a) (mata ciliar), b) (mata semidecídua), c) (cerradão), d) (cerrado sentido restrito) e e (parque cerrado); e) das áreas com amostragens não sistemáticas: f) (mata calcária), g) (campo rupestre) e h) (cerrado rupestre).

Figure 2. Photos of the study areas sampled in a systematic basis: a) (riparian forest), b) (semi-deciduous forest), c) (Cerrado), d) (cerrado sentido restrito) and e) (parque cerrado) and areas with sporadic sampling effort: f) (limestone forest), g) (campo rupestre) and h) (cerrado rupestre). 
para esse trabalho e as espécies registradas não fizeram parte de nenhuma análise.

Os indivíduos coletados em estudos anteriores (Silva \& Oniki 1988, Willis \& Oniki 1990) e os eventualmente coletados no presente trabalho estão depositados na coleção de vertebrados da Universidade Federal de Mato Grosso.

As espécies de aves foram identificadas com auxílio de binóculos $(8 \times 42)$ e gravações de vocalizações, utilizando um gravador portátil de fita cassete e microfone direcional. Foram consultados guias de campo (Ridgely \& Tudor 1989, 1994, Souza 1998, FergussonLees et al. 2000) e CDs com gravações de vozes para a identificação das espécies (Vielliard 1995a, b, 1999).

Para verificar a eficiência da amostragem na coleta de dados foram confeccionadas curvas de acumulação de espécies. Os dados de riqueza de espécies foram testados quanto a homocedasticidade, normalidade e linearidade dos dados. Como a distribuição dos dados foi normal e não homocedástica, foi utilizado o teste não paramétrico Kruskall-Wallys para verificar possíveis diferenças nas riquezas entre as cinco fitofisionomias. Após isso, o teste a posteriori de Tukey foi utilizado para se determinar quais são os pares de médias diferentes. Utilizou-se o programa R (R Development... 2011) para realizar os testes estatísticos. Um cluster com o objetivo de analisar a similaridade entre as fitofisionomias foi gerado a partir do programa MultiVariate Statistical Package - MVSP.

A ordem filogenética e nomenclatura científica adotadas na lista final estão de acordo com o proposto pelo Comitê Brasileiro de Registros Ornitológicos (2011). As espécies foram classificadas em endêmicas do Cerrado (Silva 1995, 1997, Cavalcanti 1999, Silva \& Santos 2005) e em ameaçadas de extinção (Instituto... 2005).

\section{Resultados}

A lista da avifauna da ESEC Serra das Araras é composta por 431 espécies (spp) de aves distribuídas em 295 gêneros, 63 famílias e 26 ordens. Desse total, 396 spp foram registradas de forma sistemática, $29 \mathrm{spp}$ foram registradas exclusivamente durante o esforço não sistemático e 6 spp não foram registradas nesse estudo, porém foram listadas anteriormente por Silva \& Oniki (1988) e Willis \& Oniki (1990), os quais registraram juntos 305 espécies (Tabela 1).

Com relação à distribuição da avifauna entre as diferentes fitofisionomias, na mata ciliar foi registrado um total de 256 espécies, 218 spp no cerradão, 206 spp no cerrado sentido restrito, 176 spp no parque cerrado e $150 \mathrm{spp}$ na mata semidecícua (Tabela 1).

A fitofisionomia com maior número de espécies exclusivas foi a Mata Ciliar, com 44 espécies; em seguida fica o parque cerrado com $11 \mathrm{spp}$, cerrado sentido restrito com 9 spp, a mata semidecídua com 8 spp e apenas 2 espécies foram registradas exclusivamente no cerradão.

Treze das espécies registradas na EESA são endêmicas do bioma Cerrado: Penelope ochrogaster, Columbina cyanopis, Alipiopsitta xanthops, Herpsilochmus longirostris, Melanopareia torquata, Hylocryptus rectirostris, Antilophia galeata, Suiriri islerorum, Cyanocorax cristatellus, Saltatricula atricollis, Porphyrospiza caerulescens, Charitospiza eucosma e Basileuterus leucophrys (Silva 1995, 1997, Cavalcanti 1999, Silva \& Santos 2005). Além disso, sete das espécies registradas na EESA estão presentes na lista brasileira de espécies ameaçadas de extinção: Penelope ochrogaster, Tigrisoma fasciatum, Urubitinga coronata, Columbina cyanopis, Anodorhynchus hyacinthinus, Culicivora caudacuta, Sporophila maximiliani (Instituto... 2005).

\section{Discussão}

O presente estudo adiciona 126 novas espécies a lista com 305 espécies de aves registradas anteriormente por Silva \& Oniki (1988) e Willis \& Oniki (1990) na EESA. Tal resultado já era esperado por Silva \& Oniki (1998). O táxon “Icterus hauxwelli” fora considerado por Willis \& Oniki (1990) como uma espécie diferente de I. croconotus, entretanto Lopes et al. (2009) considera "duvidosa" a validade do táxon. Ressalta-se que no presente trabalho foi registrado somente I. croconotus e caso I. hauxwelli torne-se válida para a região, será mais uma espécie somente com registro histórico para a EESA.

A riqueza de espécies de aves registrada nessa unidade de conservação representa mais de 50\% das aves listadas para o Cerrado (Silva \& Santos 2005) o que é uma riqueza de espécies elevada, sobretudo quando comparada à outras áreas de Cerrado (Tabela 2), a despeito das diferenças metodológicas entre os estudos.

A alta riqueza de espécies de aves obtina na EESA certamente está relacionada à variedade dos ambientes amostrados, os quais formam um gradiente em densidade e altura definido por formações campestres, savânicas e florestais. Cabe destacar também a localização da EESA em área de transição entre Cerrado, Amazônia e Pantanal. Vale ressaltar ainda o esforço amostral desse trabalho, o qual foi suficiente para uma amostragem exaustiva, conforme a tendência à estabilização das curvas do coletor observadas na Figura 3.

O teste Kruskal-Walis indicou diferenças significativas entre as riquezas de espécies entre as áreas (Kruskal-Wallis: $\mathrm{p}<0,001$; $\mathrm{H}=50.962$; g.l. =4) e o teste de Tukey indicou que a média das riquezas de espécie mensais registradas na mata-ciliar difere da de todas obtidas nas outras áreas; que a do cerrado sentido restrito é igual as do cerradão e parque cerrado e indicou também que as médias das riquezas observadas na mata semidecídua, no cerradão e no parque cerrado não diferem quando comparadas (Figura 4).

Certamente a maior riqueza de espécies de aves observada na mata ciliar se deve ao fato desta fitofisionomia possuir maior heterogeneidade espacial, e conseqüente maior quantidade de microhábitat que ambientes mais simples o que permite o estabelecimento de um maior número de espécies (MacArthur et al. 1962, August 1983, Antas 1995, Pianka, 2000). Uma maior riqueza de espécies em mata ciliar já era observada por Silva \& Oniki (1988) na ESEC Serra das Araras na década de oitenta e foi observado por Blamires et al. (2001) em Goiás, por Antas (1995) no Parque Nacional de Brasília (DF), por Purificação \& Castilho (2009) no Mato Grosso e também por Ferreira et al. (2009) em estudo realizado na RPPN Mata Samuel de Paula, Minas Gerais. Tais resultados reafirmam importância das matas-ciliares para conservação da avifauna do Cerrado, conforme já sugerido por Cavalcanti (1988).

Purificação \& Castilho (2009) no Parque Estadual da Serra Azul (MT) e Ferreira et al. (2009) na RPPN Mata Samuel de Paula (MG) também identificaram a dominância de registros de espécies exclusivas em matas ciliares no Cerrado, o que pode indicar que, além de servir como refúgio para algumas espécies de aves durante o período de estresse hídrico desse bioma, o que faz dela um hábitat chave para conservação desse grupo animal (Cavalcanti 1992) essa fitofisionomia também apresenta uma avifauna peculiar.

Quando comparadas as fitofisionomias quanto à similaridade, a avifauna da EESA forma dois grupos bem característicos, um grupo formado pelas fitofisionomias savânicas, cerrado sentido restrito e parque cerrado, com índice se similaridade de Sörensen superior a 0,8 ; e outro formado pelas fitofisionomias florestais mata ciliar e cerradão $(S=0,75)$ e essas duas fitofisionomias e a mata semidecídua $(\mathrm{S}=0,63)$ (Figura 5). A grosso modo, o mesmo foi observado por Almeida et al. (2003) quando analisa a diversidade beta em hábitats da Pré-Amazônia. 
Valadão, R.M.

Tabela 1. Lista sistemática das espécies de aves registradas na área da Estação Ecológica Serra das Araras. A nomenclatura científica e a seqüência filogenética seguem a proposta do CBRO (Comitê... 2011). Fitofisionomia: MS (mata semidecídua); MC (mata ciliar); CD (cerradão); CE (cerrado sentido restrito); PC (parque cerrado), NS (registro não sistemático: fora das áreas estudadas de forma sistemática) e RH [registros históricos: Silva \& Oniki (1988) e Willis \& Oniki (1990)]. ***AM (espécie presente na lista oficial das espécies de aves ameaçadas no Brasil) e ***EC (espécie endêmica do Cerrado).

Table 1. List of bird species registered at Serra das Araras Ecological Station. The scientific nomenclature and the phylogenetic sequence follow the proposal of CBRO (Comitê... 2011). Fitofisionomia (phytophysiognomies): MS (semi-deciduous forest), MC (riparian forest), CE (cerradão), CD (cerrado sentido restrito), PC (parque cerrado), NS (registered outside the areas studied systematically) and e RH [historical records: Silva \& Oniki (1988) and Willis \& Oniki (1990)]. ***AM (species present in the official list of threatened bird species in Brazil) and ***EC (endemic species of the Cerrado).

\begin{tabular}{|c|c|c|c|c|c|c|c|}
\hline \multirow{2}{*}{ Taxa } & \multicolumn{5}{|c|}{ Fitofisionomia } & \multirow{2}{*}{ NS } & \multirow{2}{*}{$\mathbf{R H}$} \\
\hline & MS & MC & CD & $\mathbf{C E}$ & PC & & \\
\hline \multicolumn{8}{|l|}{ Rheidae Bonaparte, 1849} \\
\hline Rhea americana (Linnaeus, 1758) & & & & & & $\mathrm{x}$ & \\
\hline \multicolumn{8}{|l|}{ Tinamidae Gray, 1840} \\
\hline Tinamus tao Temminck, 1815 & $\mathrm{x}$ & $\mathrm{x}$ & & & & & $\mathrm{x}$ \\
\hline Crypturellus soui (Hermann, 1783) & $\mathrm{x}$ & $\mathrm{x}$ & $\mathrm{x}$ & & & & $\mathrm{x}$ \\
\hline Crypturellus undulatus (Temminck, 1815) & $\mathrm{x}$ & $\mathrm{x}$ & $\mathrm{x}$ & & & & $\mathrm{x}$ \\
\hline Crypturellus parvirostris (Wagler, 1827) & & & & $\mathrm{x}$ & $\mathrm{x}$ & & $\mathrm{x}$ \\
\hline Crypturellus tataupa (Temminck, 1815) & $\mathrm{x}$ & $\mathrm{x}$ & & & & & $\mathrm{x}$ \\
\hline Rhynchotus rufescens (Temminck, 1815) & & & & $\mathrm{x}$ & $\mathrm{x}$ & & $\mathrm{x}$ \\
\hline \multicolumn{8}{|l|}{ Anhimidae Stejneger, 1885} \\
\hline Chauna torquata (Oken, 1816) & & & & & & $\mathrm{x}$ & \\
\hline \multicolumn{8}{|l|}{ Anatidae Leach, 1820} \\
\hline Dendrocygna viduata (Linnaeus, 1766) & & & & & & $\mathrm{x}$ & \\
\hline Dendrocygna autumnalis (Linnaeus, 1758) & & $\mathrm{x}$ & & & & & \\
\hline Cairina moschata (Linnaeus, 1758) & & $\mathrm{x}$ & & & & & \\
\hline Amazonetta brasiliensis (Gmelin, 1789) & & & & & & $\mathrm{x}$ & \\
\hline Netta peposaca (Vieillot, 1816) & & & & & & $\mathrm{x}$ & \\
\hline \multicolumn{8}{|l|}{ Cracidae Rafinesque, 1815} \\
\hline Ortalis canicollis (Wagler, 1830) & $\mathrm{x}$ & $\mathrm{x}$ & $\mathrm{x}$ & $\mathrm{x}$ & & & $\mathrm{x}$ \\
\hline Penelope superciliaris Temminck, 1815 & $\mathrm{x}$ & $\mathrm{x}$ & $\mathrm{x}$ & $\mathrm{x}$ & & & $\mathrm{x}$ \\
\hline Penelope ochrogaster Pelzeln, $1870 * * *$ AM,EC & & $\mathrm{x}$ & & & & & \\
\hline Aburria cujubi (Pelzeln, 1858) & & $\mathrm{x}$ & & & & & $\mathrm{x}$ \\
\hline Crax fasciolata Spix, 1825 & $\mathrm{x}$ & $\mathrm{x}$ & $\mathrm{x}$ & $\mathrm{x}$ & $\mathrm{x}$ & & $\mathrm{x}$ \\
\hline \multicolumn{8}{|l|}{ Odontophoridae Gould, 1844} \\
\hline Odontophorus gujanensis (Gmelin, 1789) & $\mathrm{x}$ & $\mathrm{x}$ & $\mathrm{x}$ & & & & $\mathrm{x}$ \\
\hline \multicolumn{8}{|l|}{ Podicipedidae Bonaparte, 1831} \\
\hline Tachybaptus dominicus (Linnaeus, 1766) & & & & & & $\mathrm{x}$ & \\
\hline Podilymbus podiceps (Linnaeus, 1758) & & & & & & $\mathrm{x}$ & \\
\hline \multicolumn{8}{|l|}{ Ciconiidae Sundevall, 1836} \\
\hline Jabiru mycteria (Lichtenstein, 1819) & & & & & & $\mathrm{x}$ & \\
\hline Mycteria americana Linnaeus, 1758 & & & & & & $\mathrm{x}$ & \\
\hline \multicolumn{8}{|l|}{ Phalacrocoracidae Reichenbach, 1849} \\
\hline Phalacrocorax brasilianus (Gmelin, 1789) & & $\mathrm{x}$ & & & & & \\
\hline \multicolumn{8}{|l|}{ Anhingidae Reichenbach, 1849} \\
\hline Anhinga anhinga (Linnaeus, 1766) & & $\mathrm{x}$ & & & & & $\mathrm{x}$ \\
\hline \multicolumn{8}{|l|}{ Ardeidae Leach, 1820} \\
\hline Tigrisoma lineatum (Boddaert, 1783) & & $\mathrm{x}$ & & & & & \\
\hline Tigrisoma fasciatum (Such, 1825) *** AM & & $\mathrm{x}$ & & & & & \\
\hline Cochlearius cochlearius (Linnaeus, 1766) & & & & & & $\mathrm{x}$ & \\
\hline Nycticorax nycticorax (Linnaeus, 1758) & & $\mathrm{x}$ & & & & & \\
\hline Butorides striata (Linnaeus, 1758) & & $\mathrm{x}$ & & & & & \\
\hline Bubulcus ibis (Linnaeus, 1758) & & & & & & $\mathrm{x}$ & \\
\hline Ardea cocoi Linnaeus, 1766 & & $\mathrm{x}$ & & & & & \\
\hline Ardea alba Linnaeus, 1758 & & $\mathrm{x}$ & & & & & \\
\hline Syrigma sibilatrix (Temminck, 1824) & & $\mathrm{x}$ & & & $\mathrm{x}$ & & \\
\hline Pilherodius pileatus (Boddaert, 1783) & & $\mathrm{x}$ & & & & & $\mathrm{x}$ \\
\hline Egretta thula (Molina, 1782) & & $\mathrm{x}$ & & & & & \\
\hline
\end{tabular}


Tabela 1. Continuação...

\begin{tabular}{|c|c|c|c|c|c|c|c|}
\hline \multirow{2}{*}{ Taxa } & \multicolumn{5}{|c|}{ Fitofisionomia } & \multirow{2}{*}{ NS } & \multirow{2}{*}{ RH } \\
\hline & MS & MC & CD & $\mathbf{C E}$ & PC & & \\
\hline \multicolumn{8}{|l|}{ Threskiornithidae Poche, 1904} \\
\hline Mesembrinibis cayennensis (Gmelin, 1789) & & $\mathrm{x}$ & & $\mathrm{x}$ & $\mathrm{x}$ & & \\
\hline Phimosus infuscatus (Lichtenstein, 1823) & & & & & $\mathrm{x}$ & & \\
\hline Theristicus caudatus (Boddaert, 1783) & & & & $\mathrm{x}$ & $\mathrm{x}$ & & \\
\hline \multicolumn{8}{|l|}{ Cathartidae Lafresnaye, 1839} \\
\hline Cathartes aura (Linnaeus, 1758) & & & & $\mathrm{x}$ & $\mathrm{x}$ & & $\mathrm{x}$ \\
\hline Cathartes burrovianus Cassin, 1845 & $\mathrm{x}$ & $\mathrm{x}$ & $\mathrm{x}$ & $\mathrm{x}$ & $\mathrm{x}$ & & \\
\hline Coragyps atratus (Bechstein, 1793) & $\mathrm{x}$ & $\mathrm{x}$ & $\mathrm{x}$ & $\mathrm{x}$ & $\mathrm{x}$ & & $\mathrm{x}$ \\
\hline Sarcoramphus papa (Linnaeus, 1758) & $\mathrm{x}$ & & & $\mathrm{x}$ & $\mathrm{x}$ & & $\mathrm{x}$ \\
\hline \multicolumn{8}{|l|}{ Accipitridae Vigors, 1824} \\
\hline Leptodon cayanensis (Latham, 1790) & $\mathrm{x}$ & $\mathrm{x}$ & & & & & \\
\hline Chondrohierax uncinatus (Temminck, 1822) & & $\mathrm{x}$ & $\mathrm{x}$ & $\mathrm{x}$ & & & \\
\hline Elanoides forficatus (Linnaeus, 1758) & & & & $\mathrm{x}$ & $\mathrm{x}$ & & $\mathrm{x}$ \\
\hline Gampsonyx swainsonii Vigors, 1825 & & & & $\mathrm{x}$ & $\mathrm{x}$ & & $\mathrm{x}$ \\
\hline Elanus leucurus (Vieillot, 1818) & & & & $\mathrm{x}$ & $\mathrm{x}$ & & \\
\hline Accipiter striatus Vieillot, 1808 & & $\mathrm{x}$ & & & & & \\
\hline Accipiter bicolor (Vieillot, 1817) & $\mathrm{x}$ & $\mathrm{x}$ & $\mathrm{x}$ & & & & \\
\hline Ictinia mississippiensis (Wilson, 1811) & & & & $\mathrm{x}$ & $\mathrm{x}$ & & \\
\hline Ictinia plumbea (Gmelin, 1788) & $\mathrm{x}$ & $\mathrm{x}$ & $\mathrm{x}$ & $\mathrm{x}$ & $\mathrm{x}$ & & \\
\hline Busarellus nigricollis (Latham, 1790) & & $\mathrm{x}$ & & & & & \\
\hline Rostrhamus sociabilis (Vieillot, 1817) & & & & & & $\mathrm{x}$ & $\mathrm{x}$ \\
\hline Geranospiza caerulescens (Vieillot, 1817) & $\mathrm{x}$ & $\mathrm{x}$ & & & & & $\mathrm{x}$ \\
\hline Heterospizias meridionalis (Latham, 1790) & $\mathrm{x}$ & $\mathrm{x}$ & & $\mathrm{x}$ & & & $\mathrm{x}$ \\
\hline Urubitinga urubitinga (Gmelin, 1788) & & $\mathrm{x}$ & & & & & \\
\hline Urubitinga coronata (Vieillot, 1817)***AM & & & & $\mathrm{x}$ & & & \\
\hline Rupornis magnirostris (Gmelin, 1788) & $\mathrm{x}$ & $\mathrm{x}$ & $\mathrm{x}$ & $\mathrm{x}$ & $\mathrm{x}$ & & $\mathrm{x}$ \\
\hline Parabuteo unicinctus (Temminck, 1824) & & & & & & $\mathrm{x}$ & \\
\hline Geranoaetus albicaudatus (Vieillot, 1816) & & & & $\mathrm{x}$ & $\mathrm{x}$ & & $\mathrm{x}$ \\
\hline Geranoaetus melanoleucus (Vieillot, 1819) & & & & & & $\mathrm{x}$ & \\
\hline Pseudastur albicollis (Latham, 1790) & $\mathrm{x}$ & $\mathrm{x}$ & & & & & $\mathrm{x}$ \\
\hline Buteo nitidus (Latham, 1790) & $\mathrm{x}$ & $\mathrm{x}$ & & & $\mathrm{x}$ & & $\mathrm{x}$ \\
\hline Buteo brachyurus Vieillot, 1816 & & & $\mathrm{x}$ & $\mathrm{x}$ & & & \\
\hline Buteo albonotatus Kaup, 1847 & & & & & & $\mathrm{x}$ & \\
\hline Harpia harpyja (Linnaeus, 1758) & $\mathrm{x}$ & & & $\mathrm{x}$ & & & \\
\hline Spizaetus tyrannus (Wied, 1820) & $\mathrm{x}$ & & & & & & \\
\hline Spizaetus melanoleucus (Vieillot, 1816) & $\mathrm{x}$ & $\mathrm{x}$ & & & & & $\mathrm{x}$ \\
\hline Spizaetus ornatus (Daudin, 1800) & $\mathrm{x}$ & $\mathrm{x}$ & & & & & $\mathrm{x}$ \\
\hline \multicolumn{8}{|l|}{ Falconidae Leach, 1820} \\
\hline Daptrius ater Vieillot, 1816 & & $\mathrm{x}$ & & & & & \\
\hline Caracara plancus (Miller, 1777) & & & & $\mathrm{x}$ & $\mathrm{x}$ & & $\mathrm{x}$ \\
\hline Milvago chimachima (Vieillot, 1816) & & & $\mathrm{x}$ & $\mathrm{x}$ & $\mathrm{x}$ & & $\mathrm{x}$ \\
\hline Herpetotheres cachinnans (Linnaeus, 1758) & $\mathrm{x}$ & $\mathrm{x}$ & $\mathrm{x}$ & $\mathrm{x}$ & $\mathrm{x}$ & & $\mathrm{x}$ \\
\hline Micrastur ruficollis (Vieillot, 1817) & $\mathrm{x}$ & $\mathrm{x}$ & & & & & $\mathrm{x}$ \\
\hline Micrastur semitorquatus (Vieillot, 1817) & & $\mathrm{x}$ & $\mathrm{x}$ & & & & $\mathrm{x}$ \\
\hline Falco sparverius Linnaeus, 1758 & & & & $\mathrm{x}$ & $\mathrm{x}$ & & $\mathrm{x}$ \\
\hline Falco rufigularis Daudin, 1800 & & & & $\mathrm{x}$ & $\mathrm{x}$ & & $\mathrm{x}$ \\
\hline Falco femoralis Temminck, 1822 & & & & $\mathrm{x}$ & $\mathrm{x}$ & & \\
\hline \multicolumn{8}{|l|}{ Eurypygidae Selby, 1840} \\
\hline Eurypyga helias (Pallas, 1781) & & $\mathrm{x}$ & $\mathrm{x}$ & & & & $\mathrm{x}$ \\
\hline \multicolumn{8}{|l|}{ Aramidae Bonaparte, 1852} \\
\hline Aramus guarauna (Linnaeus, 1766) & & & & & $\mathrm{x}$ & & \\
\hline \multicolumn{8}{|l|}{ Rallidae Rafinesque, 1815} \\
\hline Micropygia schomburgkii (Schomburgk, 1848) & & & & $\mathrm{x}$ & $\mathrm{x}$ & & $\mathrm{x}$ \\
\hline
\end{tabular}


Tabela 1. Continuação...

\begin{tabular}{|c|c|c|c|c|c|c|c|}
\hline \multirow{2}{*}{ Taxa } & \multicolumn{5}{|c|}{ Fitofisionomia } & \multirow{2}{*}{ NS } & \multirow{2}{*}{ RH } \\
\hline & MS & MC & CD & $\mathbf{C E}$ & PC & & \\
\hline Aramides cajanea (Statius Muller, 1776) & & $\mathrm{x}$ & $\mathrm{x}$ & $\mathrm{x}$ & $\mathrm{x}$ & & \\
\hline Laterallus viridis (Statius Muller, 1776) & & $\mathrm{x}$ & & & $\mathrm{x}$ & & $\mathrm{x}$ \\
\hline Laterallus melanophaius (Vieillot, 1819) & & & & & $\mathrm{x}$ & & $\mathrm{x}$ \\
\hline Porzana albicollis (Vieillot, 1819) & & & & & $\mathrm{x}$ & & \\
\hline Porphyrio martinica (Linnaeus, 1766) & & & & & & $\mathrm{x}$ & \\
\hline \multicolumn{8}{|l|}{ Cariamidae Bonaparte, 1850} \\
\hline Cariama cristata (Linnaeus, 1766) & & & & $\mathrm{x}$ & $\mathrm{x}$ & & $\mathrm{x}$ \\
\hline \multicolumn{8}{|l|}{ Charadriidae Leach, 1820} \\
\hline Vanellus cayanus (Latham, 1790) & & & & & & $\mathrm{x}$ & \\
\hline Vanellus chilensis (Molina, 1782) & & & & $\mathrm{x}$ & $\mathrm{x}$ & & \\
\hline \multicolumn{8}{|l|}{ Scolopacidae Rafinesque, 1815} \\
\hline Gallinago paraguaiae (Vieillot, 1816) & & & & & $\mathrm{x}$ & & \\
\hline Gallinago undulata (Boddaert, 1783) & & & & & & $\mathrm{x}$ & \\
\hline Tringa solitaria Wilson, 1813 & & & & & $\mathrm{x}$ & & \\
\hline \multicolumn{8}{|l|}{ Jacanidae Chenu \& Des Murs, 1854} \\
\hline Jacana jacana (Linnaeus, 1766) & & & & & & $\mathrm{x}$ & \\
\hline \multicolumn{8}{|l|}{ Columbidae Leach, 1820} \\
\hline Columbina minuta (Linnaeus, 1766) & & & & $\mathrm{x}$ & $\mathrm{x}$ & & $\mathrm{x}$ \\
\hline Columbina talpacoti (Temminck, 1811) & $\mathrm{x}$ & & $\mathrm{x}$ & $\mathrm{x}$ & $\mathrm{x}$ & & $\mathrm{x}$ \\
\hline Columbina squammata (Lesson, 1831) & & & $\mathrm{x}$ & $\mathrm{x}$ & $\mathrm{x}$ & & $\mathrm{x}$ \\
\hline Columbina cyanopis (Pelzeln, 1870)***AM, EC & & & & & & $\mathrm{x}$ & $\mathrm{x}$ \\
\hline Claravis pretiosa (Ferrari-Perez, 1886) & $\mathrm{x}$ & $\mathrm{x}$ & $\mathrm{x}$ & $\mathrm{x}$ & & & $\mathrm{x}$ \\
\hline Uropelia campestris (Spix, 1825) & & & & $\mathrm{x}$ & $\mathrm{x}$ & & $\mathrm{x}$ \\
\hline Patagioenas speciosa (Gmelin, 1789) & & $\mathrm{x}$ & & & & & $\mathrm{x}$ \\
\hline Patagioenas picazuro (Temminck, 1813) & $\mathrm{x}$ & $\mathrm{x}$ & $\mathrm{x}$ & $\mathrm{x}$ & $\mathrm{x}$ & & $\mathrm{x}$ \\
\hline Patagioenas cayennensis (Bonnaterre, 1792) & $\mathrm{x}$ & $\mathrm{x}$ & $\mathrm{x}$ & & $\mathrm{x}$ & & $\mathrm{x}$ \\
\hline Patagioenas plumbea (Vieillot, 1818) & $\mathrm{x}$ & $\mathrm{x}$ & $\mathrm{x}$ & & & & $\mathrm{x}$ \\
\hline Zenaida auriculata (Des Murs, 1847) & & & & $\mathrm{x}$ & $\mathrm{x}$ & & \\
\hline Leptotila verreauxi Bonaparte, 1855 & $\mathrm{x}$ & $\mathrm{x}$ & $\mathrm{x}$ & $\mathrm{x}$ & & & $\mathrm{x}$ \\
\hline Leptotila rufaxilla (Richard \& Bernard, 1792) & & $\mathrm{x}$ & & & & & $\mathrm{x}$ \\
\hline Geotrygon montana (Linnaeus, 1758) & $\mathrm{x}$ & $\mathrm{x}$ & & & & & $\mathrm{x}$ \\
\hline \multicolumn{8}{|l|}{ Psittacidae Rafinesque, 1815} \\
\hline Anodorhynchus hyacinthinus (Latham, 1790) ***Aм & & & & & $\mathrm{x}$ & & $\mathrm{x}$ \\
\hline Ara ararauna (Linnaeus, 1758) & & & & $\mathrm{x}$ & $\mathrm{x}$ & & $\mathrm{x}$ \\
\hline Ara chloropterus Gray, 1859 & & & & $\mathrm{x}$ & & & $\mathrm{x}$ \\
\hline Orthopsittaca manilata (Boddaert, 1783) & & & & $\mathrm{x}$ & $\mathrm{x}$ & & $\mathrm{x}$ \\
\hline Primolius maracana (Vieillot, 1816) & & & $\mathrm{x}$ & $\mathrm{x}$ & $\mathrm{x}$ & & \\
\hline Diopsittaca nobilis (Linnaeus, 1758) & & & $\mathrm{x}$ & $\mathrm{x}$ & $\mathrm{x}$ & & $\mathrm{x}$ \\
\hline Aratinga leucophthalma (Statius Muller, 1776) & $\mathrm{x}$ & $\mathrm{x}$ & $\mathrm{x}$ & $\mathrm{x}$ & $\mathrm{x}$ & & $\mathrm{x}$ \\
\hline Aratinga weddellii (Deville, 1851) & $\mathrm{x}$ & & & & & & $\mathrm{x}$ \\
\hline Aratinga aurea (Gmelin, 1788) & & & & $\mathrm{x}$ & $\mathrm{x}$ & & $\mathrm{x}$ \\
\hline Forpus xanthopterygius (Spix, 1824) & & $\mathrm{x}$ & & $\mathrm{x}$ & $\mathrm{x}$ & & \\
\hline Brotogeris chiriri (Vieillot, 1818) & $\mathrm{x}$ & $\mathrm{x}$ & $\mathrm{x}$ & $\mathrm{x}$ & $\mathrm{x}$ & & $\mathrm{x}$ \\
\hline Alipiopsitta xanthops (Spix, 1824)***EC & & & & $\mathrm{x}$ & $\mathrm{x}$ & & \\
\hline Pionus menstruus (Linnaeus, 1766) & $\mathrm{x}$ & $\mathrm{x}$ & $\mathrm{x}$ & $\mathrm{x}$ & $\mathrm{x}$ & & $\mathrm{x}$ \\
\hline Amazona amazonica (Linnaeus, 1766) & & $\mathrm{x}$ & $\mathrm{x}$ & $\mathrm{x}$ & $\mathrm{x}$ & & \\
\hline Amazona aestiva (Linnaeus, 1758) & $\mathrm{x}$ & $\mathrm{x}$ & & $\mathrm{x}$ & $\mathrm{x}$ & & \\
\hline \multicolumn{8}{|l|}{ Cuculidae Leach, 1820} \\
\hline Coсcусиа minuta (Vieillot, 1817) & & $\mathrm{x}$ & & & & & $\mathrm{x}$ \\
\hline Piaya cayana (Linnaeus, 1766) & $\mathrm{x}$ & $\mathrm{x}$ & $\mathrm{x}$ & $\mathrm{x}$ & $\mathrm{x}$ & & $\mathrm{x}$ \\
\hline Crotophaga major Gmelin, 1788 & & $\mathrm{x}$ & & & & & \\
\hline Crotophaga ani Linnaeus, 1758 & & & & $\mathrm{x}$ & $\mathrm{x}$ & & $\mathrm{x}$ \\
\hline Guira guira (Gmelin, 1788) & & & & $\mathrm{x}$ & $\mathrm{x}$ & & $\mathrm{x}$ \\
\hline
\end{tabular}


Tabela 1. Continuação...

\begin{tabular}{|c|c|c|c|c|c|c|c|}
\hline \multirow{2}{*}{ Taxa } & \multicolumn{5}{|c|}{ Fitofisionomia } & \multirow{2}{*}{ NS } & \multirow{2}{*}{ RH } \\
\hline & MS & MC & CD & $\mathbf{C E}$ & PC & & \\
\hline Tapera naevia (Linnaeus, 1766) & $\mathrm{x}$ & $\mathrm{x}$ & $\mathrm{x}$ & $\mathrm{x}$ & $\mathrm{x}$ & & $\mathrm{x}$ \\
\hline Dromococcyx phasianellus (Spix, 1824) & & & $\mathrm{x}$ & & & & $\mathrm{x}$ \\
\hline Dromococcyx pavoninus Pelzeln, 1870 & & $\mathrm{x}$ & $\mathrm{x}$ & & & & $\mathrm{x}$ \\
\hline \multicolumn{8}{|l|}{ Tytonidae Mathews, 1912} \\
\hline Tyto alba (Scopoli, 1769) & & & & $\mathrm{x}$ & $\mathrm{x}$ & & $\mathrm{x}$ \\
\hline \multicolumn{8}{|l|}{ Strigidae Leach, 1820} \\
\hline Megascops choliba (Vieillot, 1817) & $\mathrm{x}$ & $\mathrm{x}$ & $\mathrm{x}$ & & & & $\mathrm{x}$ \\
\hline Megascops usta (Sclater, 1858) & $\mathrm{x}$ & $\mathrm{x}$ & $\mathrm{x}$ & & & & \\
\hline Pulsatrix perspicillata (Latham, 1790) & $\mathrm{x}$ & $\mathrm{x}$ & $\mathrm{x}$ & & & & $\mathrm{x}$ \\
\hline Bubo virginianus (Gmelin, 1788) & & & & $\mathrm{x}$ & $\mathrm{x}$ & & \\
\hline Strix virgata (Cassin, 1849) & $\mathrm{x}$ & $\mathrm{x}$ & & & & & \\
\hline Strix huhula Daudin, 1800 & $\mathrm{x}$ & $\mathrm{x}$ & $\mathrm{x}$ & & & & \\
\hline Glaucidium brasilianum (Gmelin, 1788) & $\mathrm{x}$ & $\mathrm{x}$ & $\mathrm{x}$ & $\mathrm{x}$ & $\mathrm{x}$ & & $\mathrm{x}$ \\
\hline Athene cunicularia (Molina, 1782) & & & & $\mathrm{x}$ & $\mathrm{x}$ & & $\mathrm{x}$ \\
\hline Asio clamator (Vieillot, 1808) & & & $\mathrm{x}$ & $\mathrm{x}$ & & & \\
\hline Asio stygius (Wagler, 1832) & & & & & & $\mathrm{x}$ & \\
\hline \multicolumn{8}{|l|}{ Nyctibiidae Chenu \& Des Murs, 1851} \\
\hline Nyctibius grandis (Gmelin, 1789) & $\mathrm{x}$ & $\mathrm{x}$ & & $\mathrm{x}$ & & & $\mathrm{x}$ \\
\hline Nyctibius griseus (Gmelin, 1789) & $\mathrm{x}$ & $\mathrm{x}$ & & & $\mathrm{x}$ & & \\
\hline \multicolumn{8}{|l|}{ Caprimulgidae Vigors, 1825} \\
\hline Antrostomus rufus (Boddaert, 1783) & $\mathrm{x}$ & & $\mathrm{x}$ & $\mathrm{x}$ & & & $\mathrm{x}$ \\
\hline Hydropsalis albicollis (Gmelin, 1789) & & & & $\mathrm{x}$ & $\mathrm{x}$ & & $\mathrm{x}$ \\
\hline Hydropsalis parvula (Gould, 1837) & & & $\mathrm{x}$ & $\mathrm{x}$ & $\mathrm{x}$ & & \\
\hline Hydropsalis longirostris (Bonaparte, 1825) & & & & & & $\mathrm{x}$ & \\
\hline Hydropsalis torquata (Gmelin, 1789) & & & & $\mathrm{x}$ & $\mathrm{x}$ & & $\mathrm{x}$ \\
\hline Chordeiles pusillus Gould, 1861 & & & & $\mathrm{x}$ & $\mathrm{x}$ & & \\
\hline Chordeiles nacunda (Vieillot, 1817) & & & & $\mathrm{x}$ & $\mathrm{x}$ & & \\
\hline Chordeiles minor (Forster, 1771) & & & & & $\mathrm{x}$ & & \\
\hline Chordeiles acutipennis (Hermann, 1783) & & & & $\mathrm{x}$ & $\mathrm{x}$ & & $\mathrm{x}$ \\
\hline \multicolumn{8}{|l|}{ Apodidae Olphe-Galliard, 1887} \\
\hline Cypseloides fumigatus (Streubel, 1848) & & & & $\mathrm{x}$ & & & $\mathrm{x}$ \\
\hline Streptoprocne zonaris (Shaw, 1796) & $\mathrm{x}$ & & & $\mathrm{x}$ & $\mathrm{x}$ & & $\mathrm{x}$ \\
\hline Streptoprocne biscutata (Sclater, 1866) & & & & & & $\mathrm{x}$ & \\
\hline Chaetura chapmani Hellmayr, 1907 & & & & & & & $\mathrm{x}$ \\
\hline Chaetura meridionalis Hellmayr, 1907 & & & $\mathrm{x}$ & $\mathrm{x}$ & $\mathrm{x}$ & & $\mathrm{x}$ \\
\hline Tachornis squamata (Cassin, 1853) & & & & & $\mathrm{x}$ & & $\mathrm{x}$ \\
\hline \multicolumn{8}{|l|}{ Trochilidae Vigors, 1825} \\
\hline Glaucis hirsutus (Gmelin, 1788) & & $\mathrm{x}$ & $\mathrm{x}$ & $\mathrm{x}$ & & & $\mathrm{x}$ \\
\hline Phaethornis nattereri Berlepsch, 1887 & & $\mathrm{x}$ & $\mathrm{x}$ & & & & $\mathrm{x}$ \\
\hline Phaethornis ruber (Linnaeus, 1758) & & & $\mathrm{x}$ & & & & \\
\hline Phaethornis subochraceus Todd, 1915 & & $\mathrm{x}$ & $\mathrm{x}$ & & & & $\mathrm{x}$ \\
\hline Phaethornis pretrei (Lesson \& Delattre, 1839) & $\mathrm{x}$ & $\mathrm{x}$ & $\mathrm{x}$ & $\mathrm{x}$ & $\mathrm{x}$ & & $\mathrm{x}$ \\
\hline Eupetomena macroura (Gmelin, 1788) & $\mathrm{x}$ & $\mathrm{x}$ & $\mathrm{x}$ & $\mathrm{x}$ & $\mathrm{x}$ & & $\mathrm{x}$ \\
\hline Colibri serrirostris (Vieillot, 1816) & & & $\mathrm{x}$ & $\mathrm{x}$ & $\mathrm{x}$ & & \\
\hline Anthracothorax nigricollis (Vieillot, 1817) & & $\mathrm{x}$ & $\mathrm{x}$ & $\mathrm{x}$ & & & $\mathrm{x}$ \\
\hline Chrysolampis mosquitus (Linnaeus, 1758) & & $\mathrm{x}$ & & $\mathrm{x}$ & & & \\
\hline Chlorostilbon lucidus (Shaw, 1812) & & & $\mathrm{x}$ & $\mathrm{x}$ & $\mathrm{x}$ & & $\mathrm{x}$ \\
\hline Thalurania furcata (Gmelin, 1788) & $\mathrm{x}$ & $\mathrm{x}$ & $\mathrm{x}$ & & & & $\mathrm{x}$ \\
\hline Hylocharis chrysura (Shaw, 1812) & & & & $\mathrm{x}$ & $\mathrm{x}$ & & \\
\hline Polytmus guainumbi (Pallas, 1764) & & $\mathrm{x}$ & & & $\mathrm{x}$ & & \\
\hline Amazilia fimbriata (Gmelin, 1788) & $\mathrm{x}$ & $\mathrm{x}$ & $\mathrm{x}$ & $\mathrm{x}$ & $\mathrm{x}$ & & $\mathrm{x}$ \\
\hline Heliactin bilophus (Temminck, 1820) & & & & $\mathrm{x}$ & $\mathrm{x}$ & & $\mathrm{x}$ \\
\hline Heliomaster longirostris (Audebert \& Vieillot, 1801) & $\mathrm{x}$ & $\mathrm{x}$ & $\mathrm{x}$ & $\mathrm{x}$ & $\mathrm{x}$ & & $\mathrm{x}$ \\
\hline
\end{tabular}


Tabela 1. Continuação...

\begin{tabular}{|c|c|c|c|c|c|c|c|}
\hline \multirow{2}{*}{ Taxa } & \multicolumn{5}{|c|}{ Fitofisionomia } & \multirow{2}{*}{ NS } & \multirow{2}{*}{ RH } \\
\hline & MS & MC & CD & $\mathbf{C E}$ & PC & & \\
\hline Heliomaster furcifer (Shaw, 1812) & & & $\mathrm{x}$ & $\mathrm{x}$ & $\mathrm{x}$ & & \\
\hline Calliphlox amethystina (Boddaert, 1783) & & & & $\mathrm{x}$ & $\mathrm{x}$ & & \\
\hline \multicolumn{8}{|l|}{ Trogonidae Lesson, 1828} \\
\hline Trogon melanurus Swainson, 1838 & $\mathrm{x}$ & $\mathrm{x}$ & & & & & \\
\hline Trogon viridis Linnaeus, 1766 & $\mathrm{x}$ & $\mathrm{x}$ & $\mathrm{x}$ & & & & $\mathrm{x}$ \\
\hline Trogon curucui Linnaeus, 1766 & & $\mathrm{x}$ & $\mathrm{x}$ & & & & $\mathrm{x}$ \\
\hline Trogon collaris Vieillot, 1817 & $\mathrm{x}$ & $\mathrm{x}$ & $\mathrm{x}$ & & & & \\
\hline \multicolumn{8}{|l|}{ Alcedinidae Rafinesque, 1815} \\
\hline Megaceryle torquata (Linnaeus, 1766) & & $\mathrm{x}$ & & & & & $\mathrm{x}$ \\
\hline Chloroceryle amazona (Latham, 1790) & & $\mathrm{x}$ & & & & & $\mathrm{x}$ \\
\hline Chloroceryle aenea (Pallas, 1764) & & $\mathrm{x}$ & & & & & $\mathrm{x}$ \\
\hline Chloroceryle americana (Gmelin, 1788) & & $\mathrm{x}$ & & & & & $\mathrm{x}$ \\
\hline Chloroceryle inda (Linnaeus, 1766) & & $\mathrm{x}$ & & & & & $\mathrm{x}$ \\
\hline \multicolumn{8}{|l|}{ Momotidae Gray, 1840} \\
\hline Electron platyrhynchum (Leadbeater, 1829) & $\mathrm{x}$ & & & & & & $\mathrm{x}$ \\
\hline Momotus momota (Linnaeus, 1766) & $\mathrm{x}$ & $\mathrm{x}$ & $\mathrm{x}$ & & & & $\mathrm{x}$ \\
\hline \multicolumn{8}{|l|}{ Galbulidae Vigors, 1825} \\
\hline Brachygalba lugubris (Swainson, 1838) & $\mathrm{x}$ & $\mathrm{x}$ & $\mathrm{x}$ & & & & $\mathrm{x}$ \\
\hline Galbula ruficauda Cuvier, 1816 & $\mathrm{x}$ & $\mathrm{x}$ & $\mathrm{x}$ & & $\mathrm{x}$ & & $\mathrm{x}$ \\
\hline \multicolumn{8}{|l|}{ Bucconidae Horsfield, 1821} \\
\hline Notharchus macrorhynchos (Gmelin, 1788) & $\mathrm{x}$ & & & & & & $\mathrm{x}$ \\
\hline Notharchus tectus (Boddaert, 1783) & & & & $\mathrm{x}$ & & & $\mathrm{x}$ \\
\hline Bucco tamatia Gmelin, 1788 & $\mathrm{x}$ & $\mathrm{x}$ & & $\mathrm{x}$ & & & \\
\hline Nystalus striolatus (Pelzeln, 1856) & $\mathrm{x}$ & $\mathrm{x}$ & $\mathrm{x}$ & & & & \\
\hline Nystalus chacuru (Vieillot, 1816) & & & & $\mathrm{x}$ & $\mathrm{x}$ & & $\mathrm{x}$ \\
\hline Nystalus maculatus (Gmelin, 1788) & & & & $\mathrm{x}$ & $\mathrm{x}$ & & \\
\hline Nonnula ruficapilla (Tschudi, 1844) & & & & $\mathrm{x}$ & & & \\
\hline Monasa nigrifrons (Spix, 1824) & $\mathrm{x}$ & $\mathrm{x}$ & $\mathrm{x}$ & & & & $\mathrm{x}$ \\
\hline Monasa morphoeus (Hahn \& Küster, 1823) & & & & & & & $\mathrm{x}$ \\
\hline Chelidoptera tenebrosa (Pallas, 1782) & & $\mathrm{x}$ & $\mathrm{x}$ & & $\mathrm{x}$ & & $\mathrm{x}$ \\
\hline \multicolumn{8}{|l|}{ Ramphastidae Vigors, 1825} \\
\hline Ramphastos toco Statius Muller, 1776 & & $\mathrm{x}$ & & $\mathrm{x}$ & $\mathrm{x}$ & & $\mathrm{x}$ \\
\hline Ramphastos vitellinus Lichtenstein, 1823 & $\mathrm{x}$ & $\mathrm{x}$ & & & & & $\mathrm{x}$ \\
\hline Pteroglossus inscriptus Swainson, 1822 & & $\mathrm{x}$ & $\mathrm{x}$ & & & & $\mathrm{x}$ \\
\hline Pteroglossus bitorquatus Vigors, 1826 & & $\mathrm{x}$ & & & & & $\mathrm{x}$ \\
\hline Pteroglossus castanotis Gould, 1834 & $\mathrm{x}$ & $\mathrm{x}$ & $\mathrm{x}$ & $\mathrm{x}$ & $\mathrm{x}$ & & $\mathrm{x}$ \\
\hline \multicolumn{8}{|l|}{ Picidae Leach, 1820} \\
\hline Picumnus albosquamatus d'Orbigny, 1840 & $\mathrm{x}$ & $\mathrm{x}$ & $\mathrm{x}$ & & & & $\mathrm{x}$ \\
\hline Melanerpes candidus (Otto, 1796) & $\mathrm{x}$ & $\mathrm{x}$ & $\mathrm{x}$ & $\mathrm{x}$ & $\mathrm{x}$ & & \\
\hline Melanerpes cruentatus (Boddaert, 1783) & $\mathrm{x}$ & $\mathrm{x}$ & $\mathrm{x}$ & $\mathrm{x}$ & & & $\mathrm{x}$ \\
\hline Veniliornis passerinus (Linnaeus, 1766) & $\mathrm{x}$ & $\mathrm{x}$ & & & & & $\mathrm{x}$ \\
\hline Veniliornis mixtus (Boddaert, 1783) & $\mathrm{x}$ & & & & & & $\mathrm{x}$ \\
\hline Piculus leucolaemus (Natterer \& Malherbe, 1845) & $\mathrm{x}$ & & & & & & \\
\hline Piculus chrysochloros (Vieillot, 1818) & $\mathrm{x}$ & $\mathrm{x}$ & $\mathrm{x}$ & & & & $\mathrm{x}$ \\
\hline Colaptes melanochloros (Gmelin, 1788) & $\mathrm{x}$ & $\mathrm{x}$ & & $\mathrm{x}$ & & & $\mathrm{x}$ \\
\hline Colaptes campestris (Vieillot, 1818) & & & & $\mathrm{x}$ & $\mathrm{x}$ & & $\mathrm{x}$ \\
\hline Celeus elegans (Statius Muller, 1776) & $\mathrm{x}$ & $\mathrm{x}$ & $\mathrm{x}$ & & & & $\mathrm{x}$ \\
\hline Celeus lugubris (Malherbe, 1851) & $\mathrm{x}$ & $\mathrm{x}$ & $\mathrm{x}$ & & & & $\mathrm{x}$ \\
\hline Dryocopus lineatus (Linnaeus, 1766) & $\mathrm{x}$ & $\mathrm{x}$ & $\mathrm{x}$ & & & & $\mathrm{x}$ \\
\hline Campephilus rubricollis (Boddaert, 1783) & $\mathrm{x}$ & $\mathrm{x}$ & $\mathrm{x}$ & & & & $\mathrm{x}$ \\
\hline Campephilus melanoleucos (Gmelin, 1788) & $\mathrm{x}$ & $\mathrm{x}$ & $\mathrm{x}$ & $\mathrm{x}$ & & & $\mathrm{x}$ \\
\hline \multicolumn{8}{|l|}{ Thamnophilidae Swainson, 1824} \\
\hline Microrhopias quixensis (Cornalia, 1849) & & $\mathrm{x}$ & $\mathrm{x}$ & & & & $\mathrm{x}$ \\
\hline
\end{tabular}


Tabela 1. Continuação...

\begin{tabular}{|c|c|c|c|c|c|c|c|}
\hline \multirow{2}{*}{ Taxa } & \multicolumn{5}{|c|}{ Fitofisionomia } & \multirow{2}{*}{ NS } & \multirow{2}{*}{ RH } \\
\hline & MS & MC & CD & $\mathbf{C E}$ & PC & & \\
\hline Myrmeciza atrothorax (Boddaert, 1783) & & $\mathrm{x}$ & $\mathrm{x}$ & & & & $\mathrm{x}$ \\
\hline Epinecrophylla haematonota (Sclater, 1857) & & $\mathrm{x}$ & & & & & \\
\hline Myrmotherula hauxwelli (Sclater, 1857) & $\mathrm{x}$ & & & & & & $\mathrm{x}$ \\
\hline Formicivora grisea (Boddaert, 1783) & & $\mathrm{x}$ & $\mathrm{x}$ & $\mathrm{x}$ & & & $\mathrm{x}$ \\
\hline Formicivora rufa (Wied, 1831) & & $\mathrm{x}$ & $\mathrm{x}$ & $\mathrm{x}$ & & & $\mathrm{x}$ \\
\hline Dysithamnus mentalis (Temminck, 1823) & $\mathrm{x}$ & $\mathrm{x}$ & $\mathrm{x}$ & & & & $\mathrm{x}$ \\
\hline Herpsilochmus longirostris Pelzeln, 1868***EC & & $\mathrm{x}$ & $\mathrm{x}$ & & & & \\
\hline Thamnophilus doliatus (Linnaeus, 1764) & $\mathrm{x}$ & & $\mathrm{x}$ & $\mathrm{x}$ & $\mathrm{x}$ & & $\mathrm{x}$ \\
\hline Thamnophilus torquatus Swainson, 1825 & & & $\mathrm{x}$ & $\mathrm{x}$ & $\mathrm{x}$ & & $\mathrm{x}$ \\
\hline Thamnophilus punctatus (Shaw, 1809) & & $\mathrm{x}$ & $\mathrm{x}$ & & & & $\mathrm{x}$ \\
\hline Thamnophilus pelzelni Hellmayr, 1924 & $\mathrm{x}$ & $\mathrm{x}$ & $\mathrm{x}$ & & & & \\
\hline Thamnophilus caerulescens Vieillot, 1816 & & & & & & & $\mathrm{x}$ \\
\hline Cymbilaimus lineatus (Leach, 1814) & & & & & & & $\mathrm{x}$ \\
\hline Taraba major (Vieillot, 1816) & $\mathrm{x}$ & $\mathrm{x}$ & $\mathrm{x}$ & $\mathrm{x}$ & & & $\mathrm{x}$ \\
\hline Hypocnemoides maculicauda (Pelzeln, 1868) & & $\mathrm{x}$ & $\mathrm{x}$ & & & & $\mathrm{x}$ \\
\hline Pyriglena leuconota (Spix, 1824) & & $\mathrm{x}$ & $\mathrm{x}$ & & & & $\mathrm{x}$ \\
\hline Myrmoborus myotherinus (Spix, 1825) & & $\mathrm{x}$ & & & & & $\mathrm{x}$ \\
\hline Cercomacra nigrescens (Cabanis \& Heine, 1859) & & $\mathrm{x}$ & $\mathrm{x}$ & & & & $\mathrm{x}$ \\
\hline Hypocnemis cantator (Boddaert, 1783) & $\mathrm{x}$ & $\mathrm{x}$ & $\mathrm{x}$ & & & & $\mathrm{x}$ \\
\hline Willisornis poecilinotus (Cabanis, 1847) & & $\mathrm{x}$ & & & & & $\mathrm{x}$ \\
\hline Rhegmatorhina hoffmannsi (Hellmayr, 1907) & & $\mathrm{x}$ & & & & & $\mathrm{x}$ \\
\hline \multicolumn{8}{|c|}{ Melanopareiidae Ericson, Olson, Irested, Alvarenga \& Fjeldsa, 2010} \\
\hline Melanopareia torquata (Wied, 1831)***EC & & & & $\mathrm{x}$ & $\mathrm{x}$ & & $\mathrm{x}$ \\
\hline \multicolumn{8}{|l|}{ Conopophagidae Sclater \& Salvin, 1873} \\
\hline Conopophaga lineata (Wied, 1831) & & $\mathrm{x}$ & $\mathrm{x}$ & & & & $\mathrm{x}$ \\
\hline \multicolumn{8}{|l|}{ Dendrocolaptidae Gray, 1840} \\
\hline Dendrocincla fuliginosa (Vieillot, 1818) & $\mathrm{x}$ & $\mathrm{x}$ & & & & & $\mathrm{x}$ \\
\hline Sittasomus griseicapillus (Vieillot, 1818) & $\mathrm{x}$ & $\mathrm{x}$ & $\mathrm{x}$ & & & & $\mathrm{x}$ \\
\hline Glyphorynchus spirurus (Vieillot, 1819) & $\mathrm{x}$ & $\mathrm{x}$ & $\mathrm{x}$ & $\mathrm{x}$ & & & $\mathrm{x}$ \\
\hline Xiphorhynchus guttatus (Lichtenstein, 1820) & $\mathrm{x}$ & $\mathrm{x}$ & & & & & $\mathrm{x}$ \\
\hline Dendroplex picus (Gmelin, 1788) & $\mathrm{x}$ & & $\mathrm{x}$ & & & & $\mathrm{x}$ \\
\hline Lepidocolaptes angustirostris (Vieillot, 1818) & & & $\mathrm{x}$ & $\mathrm{x}$ & $\mathrm{x}$ & & $\mathrm{x}$ \\
\hline Lepidocolaptes albolineatus (Lafresnaye, 1845) & $\mathrm{x}$ & $\mathrm{x}$ & & $\mathrm{x}$ & & & $\mathrm{x}$ \\
\hline Dendrocolaptes platyrostris Spix, 1825 & $\mathrm{x}$ & $\mathrm{x}$ & $\mathrm{x}$ & & & & $\mathrm{x}$ \\
\hline Hylexetastes perrotii (Lafresnaye, 1844) & & $\mathrm{x}$ & & & & & $\mathrm{x}$ \\
\hline \multicolumn{8}{|l|}{ Furnariidae Gray, 1840} \\
\hline Xenops rutilans Temminck, 1821 & $\mathrm{x}$ & $\mathrm{x}$ & $\mathrm{x}$ & & & & $\mathrm{x}$ \\
\hline Furnarius rufus (Gmelin, 1788) & & & $\mathrm{x}$ & $\mathrm{x}$ & $\mathrm{x}$ & & $\mathrm{x}$ \\
\hline Lochmias nematura (Lichtenstein, 1823) & $\mathrm{x}$ & $\mathrm{x}$ & & & & & \\
\hline Hylocryptus rectirostris (Wied, 1831)***EC & & $\mathrm{x}$ & $\mathrm{x}$ & & & & \\
\hline Syndactyla dimidiata (Pelzeln, 1859) & $\mathrm{x}$ & & & & & & $\mathrm{x}$ \\
\hline Phacellodomus rufifrons (Wied, 1821) & & & & & $\mathrm{x}$ & & \\
\hline Phacellodomus ruber (Vieillot, 1817) & & $\mathrm{x}$ & & & $\mathrm{x}$ & & \\
\hline Synallaxis frontalis Pelzeln, 1859 & & & $\mathrm{x}$ & $\mathrm{x}$ & $\mathrm{x}$ & & $\mathrm{x}$ \\
\hline Synallaxis albescens Temminck, 1823 & & & $\mathrm{x}$ & $\mathrm{x}$ & $\mathrm{x}$ & & $\mathrm{x}$ \\
\hline Synallaxis rutilans Temminck, 1823 & $\mathrm{x}$ & & & & $\mathrm{x}$ & & $\mathrm{x}$ \\
\hline Synallaxis gujanensis (Gmelin, 1789) & & & $\mathrm{x}$ & $\mathrm{x}$ & & & $\mathrm{x}$ \\
\hline Cranioleuca vulpina (Pelzeln, 1856) & $\mathrm{x}$ & $\mathrm{x}$ & $\mathrm{x}$ & & & & $\mathrm{x}$ \\
\hline \multicolumn{8}{|l|}{ Pipridae Rafinesque, 1815} \\
\hline Neopelma pallescens (Lafresnaye, 1853) & $\mathrm{x}$ & $\mathrm{x}$ & $\mathrm{x}$ & & & & $\mathrm{x}$ \\
\hline Tyranneutes stolzmanni (Hellmayr, 1906) & & $\mathrm{x}$ & & & & & $\mathrm{x}$ \\
\hline Pipra fasciicauda Hellmayr, 1906 & $\mathrm{x}$ & $\mathrm{x}$ & $\mathrm{x}$ & & & & $\mathrm{x}$ \\
\hline Manacus manacus (Linnaeus, 1766) & & $\mathrm{x}$ & $\mathrm{x}$ & & & & $\mathrm{x}$ \\
\hline
\end{tabular}


Tabela 1. Continuação...

\begin{tabular}{|c|c|c|c|c|c|c|c|}
\hline \multirow{2}{*}{ Taxa } & \multicolumn{5}{|c|}{ Fitofisionomia } & \multirow{2}{*}{ NS } & \multirow{2}{*}{ RH } \\
\hline & MS & MC & CD & $\mathbf{C E}$ & PC & & \\
\hline Machaeropterus pyrocephalus (Sclater, 1852) & $\mathrm{x}$ & $\mathrm{x}$ & $\mathrm{x}$ & & & & $\mathrm{x}$ \\
\hline Antilophia galeata (Lichtenstein, 1823) $* * * \mathrm{EC}$ & $\mathrm{x}$ & $\mathrm{x}$ & $\mathrm{x}$ & & & & $\mathrm{x}$ \\
\hline \multicolumn{8}{|l|}{ Tityridae Gray, 1840} \\
\hline Oxyruncus cristatus Swainson, 1821 & $\mathrm{x}$ & $\mathrm{x}$ & & & & & $\mathrm{x}$ \\
\hline Terenotriccus erythrurus (Cabanis, 1847) & & & & & & & $\mathrm{x}$ \\
\hline Schiffornis turdina (Wied, 1831) & & $\mathrm{x}$ & $\mathrm{x}$ & & & & $\mathrm{x}$ \\
\hline Tityra inquisitor (Lichtenstein, 1823) & $\mathrm{x}$ & $\mathrm{x}$ & $\mathrm{x}$ & $\mathrm{x}$ & & & \\
\hline Tityra cayana (Linnaeus, 1766) & $\mathrm{x}$ & $\mathrm{x}$ & $\mathrm{x}$ & & & & $\mathrm{x}$ \\
\hline Tityra semifasciata (Spix, 1825) & & $\mathrm{x}$ & & & & & $\mathrm{x}$ \\
\hline Pachyramphus viridis (Vieillot, 1816) & & $\mathrm{x}$ & $\mathrm{x}$ & & & & \\
\hline Pachyramphus polychopterus (Vieillot, 1818) & & & $\mathrm{x}$ & $\mathrm{x}$ & $\mathrm{x}$ & & $\mathrm{x}$ \\
\hline \multicolumn{8}{|l|}{ Cotingidae Bonaparte, 1849} \\
\hline Xipholena punicea (Pallas, 1764) & & $\mathrm{x}$ & $\mathrm{x}$ & & & & $\mathrm{x}$ \\
\hline Platyrinchus mystaceus Vieillot, 1818 & & $\mathrm{x}$ & $\mathrm{x}$ & & & & $\mathrm{x}$ \\
\hline Piprites chloris (Temminck, 1822) & & $\mathrm{x}$ & & & & & $\mathrm{x}$ \\
\hline \multicolumn{8}{|l|}{ Rhynchocyclidae Berlepsch, 1907} \\
\hline Mionectes oleagineus (Lichtenstein, 1823) & $\mathrm{x}$ & $\mathrm{x}$ & $\mathrm{x}$ & & & & $\mathrm{x}$ \\
\hline Leptopogon amaurocephalus Tschudi, 1846 & $\mathrm{x}$ & $\mathrm{x}$ & $\mathrm{x}$ & & & & $\mathrm{x}$ \\
\hline Corythopis delalandi (Lesson, 1830) & $\mathrm{x}$ & $\mathrm{x}$ & $\mathrm{x}$ & & & & \\
\hline Tolmomyias sulphurescens (Spix, 1825) & $\mathrm{x}$ & $\mathrm{x}$ & $\mathrm{x}$ & & & & $\mathrm{x}$ \\
\hline Todirostrum cinereum (Linnaeus, 1766) & & & $\mathrm{x}$ & $\mathrm{x}$ & $\mathrm{x}$ & & $\mathrm{x}$ \\
\hline Poecilotriccus latirostris (Pelzeln, 1868) & & $\mathrm{x}$ & $\mathrm{x}$ & & & & $\mathrm{x}$ \\
\hline Hemitriccus flammulatus Berlepsch, 1901 & & $\mathrm{x}$ & $\mathrm{x}$ & & & & \\
\hline Hemitriccus striaticollis (Lafresnaye, 1853) & & $\mathrm{x}$ & $\mathrm{x}$ & & & & $\mathrm{x}$ \\
\hline Hemitriccus margaritaceiventer (d'Orbigny \& Lafresnaye, 1837) & & $\mathrm{x}$ & $\mathrm{x}$ & $\mathrm{x}$ & $\mathrm{x}$ & & $\mathrm{x}$ \\
\hline \multicolumn{8}{|l|}{ Tyrannidae Vigors, 1825} \\
\hline Hirundinea ferruginea (Gmelin, 1788) & & & & $\mathrm{x}$ & & & $\mathrm{x}$ \\
\hline Euscarthmus meloryphus Wied, 1831 & & & & $\mathrm{x}$ & & & $\mathrm{x}$ \\
\hline Euscarthmus rufomarginatus (Pelzeln, 1868) & & & & & & $\mathrm{x}$ & $\mathrm{x}$ \\
\hline Camptostoma obsoletum (Temminck, 1824) & & $\mathrm{x}$ & $\mathrm{x}$ & $\mathrm{x}$ & $\mathrm{x}$ & & $\mathrm{x}$ \\
\hline Elaenia flavogaster (Thunberg, 1822) & & & $\mathrm{x}$ & $\mathrm{x}$ & $\mathrm{x}$ & & $\mathrm{x}$ \\
\hline Elaenia albiceps (d’Orbigny \& Lafresnaye, 1837) & & $\mathrm{x}$ & $\mathrm{x}$ & & & & $\mathrm{x}$ \\
\hline Elaenia parvirostris Pelzeln, 1868 & & & $\mathrm{x}$ & $\mathrm{x}$ & $\mathrm{x}$ & & $\mathrm{x}$ \\
\hline Elaenia cristata Pelzeln, 1868 & & & $\mathrm{x}$ & $\mathrm{x}$ & $\mathrm{x}$ & & $\mathrm{x}$ \\
\hline Elaenia chiriquensis Lawrence, 1865 & & & & $\mathrm{x}$ & $\mathrm{x}$ & & $\mathrm{x}$ \\
\hline Suiriri suiriri (Vieillot, 1818) & & $\mathrm{x}$ & $\mathrm{x}$ & $\mathrm{x}$ & $\mathrm{x}$ & & $\mathrm{x}$ \\
\hline Suiriri islerorum Zimmer, Whittaker \& Oren, $2001 * * * \mathrm{EC}$ & & $\mathrm{x}$ & $\mathrm{x}$ & $\mathrm{x}$ & $\mathrm{x}$ & & \\
\hline Myiopagis gaimardii (d’Orbigny, 1839) & & $\mathrm{x}$ & $\mathrm{x}$ & & & & $\mathrm{x}$ \\
\hline Myiopagis caniceps (Swainson, 1835) & & $\mathrm{x}$ & $\mathrm{x}$ & & & & $\mathrm{x}$ \\
\hline Myiopagis viridicata (Vieillot, 1817) & $\mathrm{x}$ & $\mathrm{x}$ & $\mathrm{x}$ & & & & $\mathrm{x}$ \\
\hline Phaeomyias murina (Spix, 1825) & & & $\mathrm{x}$ & $\mathrm{x}$ & $\mathrm{x}$ & & $\mathrm{x}$ \\
\hline Phyllomyias fasciatus (Thunberg, 1822) & & $\mathrm{x}$ & $\mathrm{x}$ & & & & $\mathrm{x}$ \\
\hline Culicivora caudacuta (Vieillot, 1818) ***AM & & & & $\mathrm{x}$ & $\mathrm{x}$ & & $\mathrm{x}$ \\
\hline Attila bolivianus Lafresnaye, 1848 & $\mathrm{x}$ & $\mathrm{x}$ & $\mathrm{x}$ & & & & $\mathrm{x}$ \\
\hline Legatus leucophaius (Vieillot, 1818) & & $\mathrm{x}$ & $\mathrm{x}$ & & & & $\mathrm{x}$ \\
\hline Ramphotrigon ruficauda (Spix, 1825) & & $\mathrm{x}$ & $\mathrm{x}$ & & & & $\mathrm{x}$ \\
\hline Myiarchus tuberculifer (d'Orbigny \& Lafresnaye, 1837) & $\mathrm{x}$ & $\mathrm{x}$ & $\mathrm{x}$ & $\mathrm{x}$ & & & $\mathrm{x}$ \\
\hline Myiarchus swainsoni Cabanis \& Heine, 1859 & & $\mathrm{x}$ & $\mathrm{x}$ & $\mathrm{x}$ & $\mathrm{x}$ & & $\mathrm{x}$ \\
\hline Myiarchus ferox (Gmelin, 1789) & & $\mathrm{x}$ & $\mathrm{x}$ & $\mathrm{x}$ & $\mathrm{x}$ & & $\mathrm{x}$ \\
\hline Myiarchus tyrannulus (Statius Muller, 1776) & & & $\mathrm{x}$ & $\mathrm{x}$ & $\mathrm{x}$ & & $\mathrm{x}$ \\
\hline Sirystes sibilator (Vieillot, 1818) & $\mathrm{x}$ & $\mathrm{x}$ & $\mathrm{x}$ & & & & $\mathrm{x}$ \\
\hline Casiornis rufus (Vieillot, 1816) & & $\mathrm{x}$ & $\mathrm{x}$ & $\mathrm{x}$ & & & $\mathrm{x}$ \\
\hline Pitangus sulphuratus (Linnaeus, 1766) & $\mathrm{x}$ & $\mathrm{x}$ & $\mathrm{x}$ & $\mathrm{x}$ & $\mathrm{x}$ & & $\mathrm{x}$ \\
\hline
\end{tabular}


Tabela 1. Continuação...

\begin{tabular}{|c|c|c|c|c|c|c|c|}
\hline \multirow{2}{*}{ Taxa } & \multicolumn{5}{|c|}{ Fitofisionomia } & \multirow{2}{*}{ NS } & \multirow{2}{*}{ RH } \\
\hline & MS & MC & CD & $\mathbf{C E}$ & PC & & \\
\hline Philohydor lictor (Lichtenstein, 1823) & & $\mathrm{x}$ & $\mathrm{x}$ & $\mathrm{x}$ & & & $\mathrm{x}$ \\
\hline Machetornis rixosa (Vieillot, 1819) & & & & $\mathrm{x}$ & $\mathrm{x}$ & & $\mathrm{x}$ \\
\hline Myiodynastes maculatus (Statius Muller, 1776) & $\mathrm{x}$ & $\mathrm{x}$ & $\mathrm{x}$ & $\mathrm{x}$ & $\mathrm{x}$ & & $\mathrm{x}$ \\
\hline Megarynchus pitangua (Linnaeus, 1766) & $\mathrm{x}$ & $\mathrm{x}$ & $\mathrm{x}$ & $\mathrm{x}$ & $\mathrm{x}$ & & $\mathrm{x}$ \\
\hline Myiozetetes cayanensis (Linnaeus, 1766) & & $\mathrm{x}$ & $\mathrm{x}$ & & & & $\mathrm{x}$ \\
\hline Tyrannus albogularis Burmeister, 1856 & $\mathrm{x}$ & $\mathrm{x}$ & $\mathrm{x}$ & $\mathrm{x}$ & $\mathrm{x}$ & & \\
\hline Tyrannus melancholicus Vieillot, 1819 & $\mathrm{x}$ & $\mathrm{x}$ & $\mathrm{x}$ & $\mathrm{x}$ & $\mathrm{x}$ & & $\mathrm{x}$ \\
\hline Tyrannus savana Vieillot, 1808 & $\mathrm{x}$ & $\mathrm{x}$ & $\mathrm{x}$ & $\mathrm{x}$ & $\mathrm{x}$ & & $\mathrm{x}$ \\
\hline Griseotyrannus aurantioatrocristatus (d'Orbigny \& Lafresnaye, 1837) & $\mathrm{x}$ & $\mathrm{x}$ & $\mathrm{x}$ & $\mathrm{x}$ & $\mathrm{x}$ & & \\
\hline Empidonomus varius (Vieillot, 1818) & $\mathrm{x}$ & $\mathrm{x}$ & $\mathrm{x}$ & $\mathrm{x}$ & $\mathrm{x}$ & & $\mathrm{x}$ \\
\hline Colonia colonus (Vieillot, 1818) & $\mathrm{x}$ & $\mathrm{x}$ & $\mathrm{x}$ & & & & $\mathrm{x}$ \\
\hline Myiophobus fasciatus (Statius Muller, 1776) & & $\mathrm{x}$ & $\mathrm{x}$ & $\mathrm{x}$ & $\mathrm{x}$ & & $\mathrm{x}$ \\
\hline Sublegatus modestus (Wied, 1831) & & & & $\mathrm{x}$ & $\mathrm{x}$ & & $\mathrm{x}$ \\
\hline Pyrocephalus rubinus (Boddaert, 1783) & & & & $\mathrm{x}$ & $\mathrm{x}$ & & \\
\hline Gubernetes yetapa (Vieillot, 1818) & & & & & $\mathrm{x}$ & & $\mathrm{x}$ \\
\hline Cnemotriccus fuscatus (Wied, 1831) & & $\mathrm{x}$ & $\mathrm{x}$ & & & & $\mathrm{x}$ \\
\hline Lathrotriccus euleri (Cabanis, 1868) & & $\mathrm{x}$ & $\mathrm{x}$ & & & & $\mathrm{x}$ \\
\hline Knipolegus lophotes Boie, 1828 & & $\mathrm{x}$ & & & & & \\
\hline Xolmis cinereus (Vieillot, 1816) & & & & $\mathrm{x}$ & $\mathrm{x}$ & & $\mathrm{x}$ \\
\hline Xolmis velatus (Lichtenstein, 1823) & & & & $\mathrm{x}$ & $\mathrm{x}$ & & \\
\hline \multicolumn{8}{|l|}{ Vireonidae Swainson, 1837} \\
\hline Cyclarhis gujanensis (Gmelin, 1789) & & & $\mathrm{x}$ & $\mathrm{x}$ & $\mathrm{x}$ & & $\mathrm{x}$ \\
\hline Vireo olivaceus (Linnaeus, 1766) & & & $\mathrm{x}$ & $\mathrm{x}$ & $\mathrm{x}$ & & $\mathrm{x}$ \\
\hline Hylophilus muscicapinus Sclater \& Salvin, 1873 & & & & & & & $\mathrm{x}$ \\
\hline \multicolumn{8}{|l|}{ Corvidae Leach, 1820} \\
\hline Cyanocorax cyanomelas (Vieillot, 1818) & $\mathrm{x}$ & $\mathrm{x}$ & $\mathrm{x}$ & $\mathrm{x}$ & & & $\mathrm{x}$ \\
\hline Cyanocorax cristatellus (Temminck, 1823)***EC & & & & $\mathrm{x}$ & $\mathrm{x}$ & & $\mathrm{x}$ \\
\hline \multicolumn{8}{|l|}{ Hirundinidae Rafinesque, 1815} \\
\hline Pygochelidon cyanoleuca (Vieillot, 1817) & & & & $\mathrm{x}$ & $\mathrm{x}$ & & \\
\hline Stelgidopteryx ruficollis (Vieillot, 1817) & & $\mathrm{x}$ & $\mathrm{x}$ & $\mathrm{x}$ & & & $\mathrm{x}$ \\
\hline Progne tapera (Vieillot, 1817) & & & & $\mathrm{x}$ & $\mathrm{x}$ & & $\mathrm{x}$ \\
\hline Progne chalybea (Gmelin, 1789) & & & & $\mathrm{x}$ & $\mathrm{x}$ & & \\
\hline Tachycineta leucorrhoa (Vieillot, 1817) & & & & $\mathrm{x}$ & $\mathrm{x}$ & & \\
\hline Hirundo rustica Linnaeus, 1758 & & & & $\mathrm{x}$ & $\mathrm{x}$ & & \\
\hline \multicolumn{8}{|l|}{ Troglodytidae Swainson, 1831} \\
\hline Troglodytes musculus Naumann, 1823 & & & & $\mathrm{x}$ & $\mathrm{x}$ & & $\mathrm{x}$ \\
\hline Campylorhynchus turdinus (Wied, 1831) & $\mathrm{x}$ & $\mathrm{x}$ & $\mathrm{x}$ & & & & $\mathrm{x}$ \\
\hline Pheugopedius genibarbis (Swainson, 1838) & & $\mathrm{x}$ & $\mathrm{x}$ & & & & $\mathrm{x}$ \\
\hline Cantorchilus leucotis (Lafresnaye, 1845) & & $\mathrm{x}$ & $\mathrm{x}$ & & & & $\mathrm{x}$ \\
\hline \multicolumn{8}{|l|}{ Donacobiidae Aleixo \& Pacheco, 2006} \\
\hline Donacobius atricapilla (Linnaeus, 1766) & & & & & & $\mathrm{x}$ & \\
\hline \multicolumn{8}{|l|}{ Polioptilidae Baird, 1858} \\
\hline Polioptila dumicola (Vieillot, 1817) & $\mathrm{x}$ & $\mathrm{x}$ & $\mathrm{x}$ & $\mathrm{x}$ & $\mathrm{x}$ & & $\mathrm{x}$ \\
\hline \multicolumn{8}{|l|}{ Turdidae Rafinesque, 1815} \\
\hline Catharus fuscescens (Stephens, 1817) & $\mathrm{x}$ & $\mathrm{x}$ & $\mathrm{x}$ & & & & $\mathrm{x}$ \\
\hline Turdus rufiventris Vieillot, 1818 & $\mathrm{x}$ & $\mathrm{x}$ & $\mathrm{x}$ & $\mathrm{x}$ & $\mathrm{x}$ & & $\mathrm{x}$ \\
\hline Turdus leucomelas Vieillot, 1818 & $\mathrm{x}$ & $\mathrm{x}$ & $\mathrm{x}$ & $\mathrm{x}$ & $\mathrm{x}$ & & $\mathrm{x}$ \\
\hline Turdus fumigatus Lichtenstein, 1823 & & $\mathrm{x}$ & $\mathrm{x}$ & & & & \\
\hline Turdus amaurochalinus Cabanis, 1850 & $\mathrm{x}$ & $\mathrm{x}$ & $\mathrm{x}$ & & & & $\mathrm{x}$ \\
\hline Turdus albicollis Vieillot, 1818 & & & & & & $\mathrm{x}$ & $\mathrm{x}$ \\
\hline \multicolumn{8}{|l|}{ Mimidae Bonaparte, 1853} \\
\hline Mimus saturninus (Lichtenstein, 1823) & & & $\mathrm{x}$ & $\mathrm{x}$ & $\mathrm{x}$ & & $\mathrm{x}$ \\
\hline Coerebidae d'Orbigny \& Lafresnaye, 1838 & & & & & & & \\
\hline
\end{tabular}


Tabela 1. Continuação...

\begin{tabular}{|c|c|c|c|c|c|c|c|}
\hline \multirow{2}{*}{ Taxa } & \multicolumn{5}{|c|}{ Fitofisionomia } & \multirow{2}{*}{ NS } & \multirow{2}{*}{ RH } \\
\hline & MS & MC & CD & $\mathbf{C E}$ & PC & & \\
\hline Coereba flaveola (Linnaeus, 1758) & $\mathrm{x}$ & $\mathrm{x}$ & $\mathrm{x}$ & $\mathrm{x}$ & $\mathrm{x}$ & & $\mathrm{x}$ \\
\hline \multicolumn{8}{|l|}{ Thraupidae Cabanis, 1847} \\
\hline Saltator maximus (Statius Muller, 1776) & $\mathrm{x}$ & $\mathrm{x}$ & $\mathrm{x}$ & & & & $\mathrm{x}$ \\
\hline Saltator coerulescens Vieillot, 1817 & $\mathrm{x}$ & $\mathrm{x}$ & $\mathrm{x}$ & $\mathrm{x}$ & $\mathrm{x}$ & & \\
\hline Saltator similis d'Orbigny \& Lafresnaye, 1837 & & $\mathrm{x}$ & $\mathrm{x}$ & $\mathrm{x}$ & & & \\
\hline Saltatricula atricollis (Vieillot, 1817)***EC & & & & $\mathrm{x}$ & $\mathrm{x}$ & & $\mathrm{x}$ \\
\hline Cypsnagra hirundinacea (Lesson, 1831) & & & $\mathrm{x}$ & $\mathrm{x}$ & $\mathrm{x}$ & & $\mathrm{x}$ \\
\hline Tachyphonus rufus (Boddaert, 1783) & & $\mathrm{x}$ & $\mathrm{x}$ & $\mathrm{x}$ & & & $\mathrm{x}$ \\
\hline Ramphocelus carbo (Pallas, 1764) & $\mathrm{x}$ & $\mathrm{x}$ & $\mathrm{x}$ & $\mathrm{x}$ & & & $\mathrm{x}$ \\
\hline Lanio luctuosus (d'Orbigny \& Lafresnaye, 1837) & & $\mathrm{x}$ & & & & & $\mathrm{x}$ \\
\hline Lanio cucullatus (Statius Muller, 1776) & & & & $\mathrm{x}$ & $\mathrm{x}$ & & $\mathrm{x}$ \\
\hline Lanio versicolor (d'Orbigny \& Lafresnaye, 1837) & & $\mathrm{x}$ & $\mathrm{x}$ & & & & $\mathrm{x}$ \\
\hline Lanio penicillatus (Spix, 1825) & $\mathrm{x}$ & $\mathrm{x}$ & $\mathrm{x}$ & & & & $\mathrm{x}$ \\
\hline Tangara mexicana (Linnaeus, 1766) & & $\mathrm{x}$ & & & & & $\mathrm{x}$ \\
\hline Tangara chilensis (Vigors, 1832) & & $\mathrm{x}$ & & & & & $\mathrm{x}$ \\
\hline Tangara sayaca (Linnaeus, 1766) & $\mathrm{x}$ & $\mathrm{x}$ & $\mathrm{x}$ & $\mathrm{x}$ & $\mathrm{x}$ & & $\mathrm{x}$ \\
\hline Tangara palmarum (Wied, 1823) & $\mathrm{x}$ & $\mathrm{x}$ & $\mathrm{x}$ & $\mathrm{x}$ & & & $\mathrm{x}$ \\
\hline Tangara cyanicollis (d'Orbigny \& Lafresnaye, 1837) & & $\mathrm{x}$ & $\mathrm{x}$ & & & & $\mathrm{x}$ \\
\hline Tangara cayana (Linnaeus, 1766) & $\mathrm{x}$ & $\mathrm{x}$ & $\mathrm{x}$ & $\mathrm{x}$ & $\mathrm{x}$ & & $\mathrm{x}$ \\
\hline Neothraupis fasciata (Lichtenstein, 1823) & & & & $\mathrm{x}$ & $\mathrm{x}$ & & $\mathrm{x}$ \\
\hline Cissopis leverianus (Gmelin, 1788) & $\mathrm{x}$ & $\mathrm{x}$ & $\mathrm{x}$ & $\mathrm{x}$ & $\mathrm{x}$ & & $\mathrm{x}$ \\
\hline Schistochlamys melanopis (Latham, 1790) & & & $\mathrm{x}$ & $\mathrm{x}$ & $\mathrm{x}$ & & $\mathrm{x}$ \\
\hline Paroaria capitata (d'Orbigny \& Lafresnaye, 1837) & & & & $\mathrm{x}$ & $\mathrm{x}$ & & $\mathrm{x}$ \\
\hline Tersina viridis (Illiger, 1811) & & $\mathrm{x}$ & $\mathrm{x}$ & $\mathrm{x}$ & & & $\mathrm{x}$ \\
\hline Dacnis lineata (Gmelin, 1789) & & $\mathrm{x}$ & $\mathrm{x}$ & $\mathrm{x}$ & & & $\mathrm{x}$ \\
\hline Dacnis cayana (Linnaeus, 1766) & $\mathrm{x}$ & $\mathrm{x}$ & $\mathrm{x}$ & $\mathrm{x}$ & $\mathrm{x}$ & & $\mathrm{x}$ \\
\hline Cyanerpes caeruleus (Linnaeus, 1758) & & $\mathrm{x}$ & $\mathrm{x}$ & $\mathrm{x}$ & $\mathrm{x}$ & & $\mathrm{x}$ \\
\hline Cyanerpes cyaneus (Linnaeus, 1766) & & $\mathrm{x}$ & $\mathrm{x}$ & $\mathrm{x}$ & & & $\mathrm{x}$ \\
\hline Chlorophanes spiza (Linnaeus, 1758) & & $\mathrm{x}$ & & & & & $\mathrm{x}$ \\
\hline Hemithraupis guira (Linnaeus, 1766) & $\mathrm{x}$ & $\mathrm{x}$ & $\mathrm{x}$ & & & & $\mathrm{x}$ \\
\hline Hemithraupis flavicollis (Vieillot, 1818) & & $\mathrm{x}$ & $\mathrm{x}$ & & & & $\mathrm{x}$ \\
\hline Conirostrum speciosum (Temminck, 1824) & & $\mathrm{x}$ & $\mathrm{x}$ & & & & \\
\hline \multicolumn{8}{|l|}{ Emberizidae Vigors, 1825} \\
\hline Zonotrichia capensis (Statius Muller, 1776) & & & & $\mathrm{x}$ & $\mathrm{x}$ & & $\mathrm{x}$ \\
\hline Ammodramus humeralis (Bosc, 1792) & & & & $\mathrm{x}$ & $\mathrm{x}$ & & $\mathrm{x}$ \\
\hline Porphyrospiza caerulescens (Wied, 1830)***EC & & & & & & $\mathrm{x}$ & $\mathrm{x}$ \\
\hline Sicalis citrina Pelzeln, 1870 & & & & $\mathrm{x}$ & $\mathrm{x}$ & & \\
\hline Sicalis flaveola (Linnaeus, 1766) & & & $\mathrm{x}$ & $\mathrm{x}$ & $\mathrm{x}$ & & $\mathrm{x}$ \\
\hline Emberizoides herbicola (Vieillot, 1817) & & & & $\mathrm{x}$ & $\mathrm{x}$ & & $\mathrm{x}$ \\
\hline Volatinia jacarina (Linnaeus, 1766) & & & & $\mathrm{x}$ & $\mathrm{x}$ & & $\mathrm{x}$ \\
\hline Sporophila plumbea (Wied, 1830) & & & $\mathrm{x}$ & $\mathrm{x}$ & $\mathrm{x}$ & & \\
\hline Sporophila collaris (Boddaert, 1783) & & & & & & $\mathrm{x}$ & \\
\hline Sporophila lineola (Linnaeus, 1758) & & & & $\mathrm{x}$ & $\mathrm{x}$ & & \\
\hline Sporophila nigricollis (Vieillot, 1823) & & & & $\mathrm{x}$ & $\mathrm{x}$ & & \\
\hline Sporophila caerulescens (Vieillot, 1823) & & & & $\mathrm{x}$ & & & $\mathrm{x}$ \\
\hline Sporophila leucoptera (Vieillot, 1817) & & & & $\mathrm{x}$ & $\mathrm{x}$ & & $\mathrm{x}$ \\
\hline Sporophila angolensis (Linnaeus, 1766) & & & $\mathrm{x}$ & $\mathrm{x}$ & & & $\mathrm{x}$ \\
\hline Sporophila maximiliani (Cabanis, 1851) $* * * \mathrm{AM}$ & & & & & & $\mathrm{x}$ & \\
\hline Tiaris fuliginosus (Wied, 1830) & & $\mathrm{x}$ & $\mathrm{x}$ & & & & \\
\hline Arremon taciturnus (Hermann, 1783) & $\mathrm{x}$ & $\mathrm{x}$ & $\mathrm{x}$ & & & & $\mathrm{x}$ \\
\hline Arremon flavirostris Swainson, 1838 & $\mathrm{x}$ & $\mathrm{x}$ & $\mathrm{x}$ & & & & \\
\hline Charitospiza eucosma Oberholser, 1905***EC & & & & $\mathrm{x}$ & & & \\
\hline Cardinalidae Ridgway, 1901 & & & & & & & \\
\hline
\end{tabular}


Tabela 1. Continuação...

\begin{tabular}{|c|c|c|c|c|c|c|c|}
\hline \multirow{2}{*}{ Taxa } & \multicolumn{5}{|c|}{ Fitofisionomia } & \multirow{2}{*}{ NS } & \multirow{2}{*}{ RH } \\
\hline & MS & MC & CD & $\mathbf{C E}$ & PC & & \\
\hline Piranga flava (Vieillot, 1822) & & & $\mathrm{x}$ & $\mathrm{x}$ & $\mathrm{x}$ & & $\mathrm{x}$ \\
\hline Cyanoloxia brissonii (Lichtenstein, 1823) & & $\mathrm{x}$ & $\mathrm{x}$ & $\mathrm{x}$ & & & $\mathrm{x}$ \\
\hline \multicolumn{8}{|c|}{$\begin{array}{l}\text { Parulidae Wetmore, Friedmann, Lincoln, Miller, Peters, van Rossem, } \\
\text { Van Tyne \& Zimmer } 1947\end{array}$} \\
\hline Parula pitiayumi (Vieillot, 1817) & $\mathrm{x}$ & $\mathrm{x}$ & $\mathrm{x}$ & & & & $\mathrm{x}$ \\
\hline Geothlypis aequinoctialis (Gmelin, 1789) & & $\mathrm{x}$ & $\mathrm{x}$ & & & & $\mathrm{x}$ \\
\hline Basileuterus hypoleucus Bonaparte, 1830 & $\mathrm{x}$ & $\mathrm{x}$ & & & & & $\mathrm{x}$ \\
\hline Basileuterus flaveolus (Baird, 1865) & $\mathrm{x}$ & $\mathrm{x}$ & $\mathrm{x}$ & & & & $\mathrm{x}$ \\
\hline Basileuterus leucophrys Pelzeln, 1868***EC & & $\mathrm{x}$ & & & & & \\
\hline \multicolumn{8}{|l|}{ Icteridae Vigors, 1825} \\
\hline Psarocolius decumanus (Pallas, 1769) & $\mathrm{x}$ & $\mathrm{x}$ & $\mathrm{x}$ & & $\mathrm{x}$ & & $\mathrm{x}$ \\
\hline Procacicus solitarius (Vieillot, 1816) & & $\mathrm{x}$ & & & & & \\
\hline Cacicus haemorrhous (Linnaeus, 1766) & & $\mathrm{x}$ & $\mathrm{x}$ & $\mathrm{x}$ & & & $\mathrm{x}$ \\
\hline Cacicus cela (Linnaeus, 1758) & $\mathrm{x}$ & $\mathrm{x}$ & $\mathrm{x}$ & $\mathrm{x}$ & $\mathrm{x}$ & & $\mathrm{x}$ \\
\hline Icterus cayanensis (Linnaeus, 1766) & & $\mathrm{x}$ & $\mathrm{x}$ & $\mathrm{x}$ & & & $\mathrm{x}$ \\
\hline Icterus croconotus (Wagler, 1829) & & & & $\mathrm{x}$ & $\mathrm{x}$ & & $\mathrm{x}$ \\
\hline Gnorimopsar chopi (Vieillot, 1819) & & & $\mathrm{x}$ & $\mathrm{x}$ & $\mathrm{x}$ & & $\mathrm{x}$ \\
\hline Molothrus oryzivorus (Gmelin, 1788) & & $\mathrm{x}$ & $\mathrm{x}$ & $\mathrm{x}$ & & & $\mathrm{x}$ \\
\hline Molothrus bonariensis (Gmelin, 1789) & $\mathrm{x}$ & $\mathrm{x}$ & & $\mathrm{x}$ & $\mathrm{x}$ & & $\mathrm{x}$ \\
\hline \multicolumn{8}{|l|}{ Fringillidae Leach, 1820} \\
\hline Euphonia chlorotica (Linnaeus, 1766) & $\mathrm{x}$ & $\mathrm{x}$ & $\mathrm{x}$ & $\mathrm{x}$ & $\mathrm{x}$ & & $\mathrm{x}$ \\
\hline Euphonia violacea (Linnaeus, 1758) & & $\mathrm{x}$ & & & & & $\mathrm{x}$ \\
\hline Euphonia laniirostris (d'Orbigny \& Lafresnaye, 1837) & $\mathrm{x}$ & $\mathrm{x}$ & $\mathrm{x}$ & $\mathrm{x}$ & & & $\mathrm{x}$ \\
\hline Euphonia rufiventris (Vieillot, 1819) & & $\mathrm{x}$ & $\mathrm{x}$ & & & & $\mathrm{x}$ \\
\hline
\end{tabular}

Tabela 2. Número de espécies de aves registradas em diferentes áreas do bioma Cerrado.

Table 2. Number of bird species registered in different areas of the Cerrado Biome.

\begin{tabular}{|c|c|c|}
\hline Local (estado) & $\mathrm{N}^{0}$ de espécies & Fonte \\
\hline Vila Bela da Santíssima Trindade (MT) & 312 & Silveira \& D’Horta (2002) \\
\hline Chapada dos Guimarães (MT) & 393 & Lopes et al. (2009) \\
\hline Parque Estadual da Serra Azul (MT) & 131 & Purificação \& Castilho (2009) \\
\hline Planalto da Bodoquena (MS) & 353 & Pivatto et al. (2006) \\
\hline ESEC Itirapina (SP) & 231 & Mota-Júnior et al. (2008) \\
\hline ESEC Caetus (SP) & 293 & Cavarzere et al. (2009) \\
\hline Três Marias (MG) & 101 & Ribon et al. (1995) \\
\hline PARNA Serra da Canastra (MG) & 282 & Silveira (1998) \\
\hline RPPN do Panga (MG) & 231 & Marçal-Júnior et al. (2006) \\
\hline Fazenda Brejão (MG) & 273 & Faria et al. (2009) \\
\hline RPPN Mata Samuel de Paula (MG) & 188 & Ferreira et al. (2009) \\
\hline PARNA Serra do Cipó (MG) & 338 & Melo-Júnior et al. (2001), Rodrigues et al. $(2005,2011)$ \\
\hline Caldazinha (GO) & 124 & Blamires et al. (2001) \\
\hline Niquelândia (GO) & 156 & Curcino et al. (2007) \\
\hline PARNA de Brasília (DF) & 265 & Antas (1995) \\
\hline ESEC Água Emendadas (DF) & 301 & Bagno (1998), Lopes et al. (2005) \\
\hline ESEC Jardim Botânico (DF) & 238 & Tubelis \& Cavalcanti (2001) \\
\hline PARNA Chapada Diamantina (BA) & 359 & Parrini et al. (1999) \\
\hline ESEC Serra Geral do Tocantins (BA/TO) & 254 & Rego et al. (2011) \\
\hline Sudeste do Estado do Tocantins (TO) & 308 & Pacheco \& Olmos (2006) \\
\hline Pedro Afonso (TO) & 254 & Lopes \& Braz (2007) \\
\hline Região do Cantão (TO) & 418 & Pinheiro \& Dornas (2009) \\
\hline
\end{tabular}




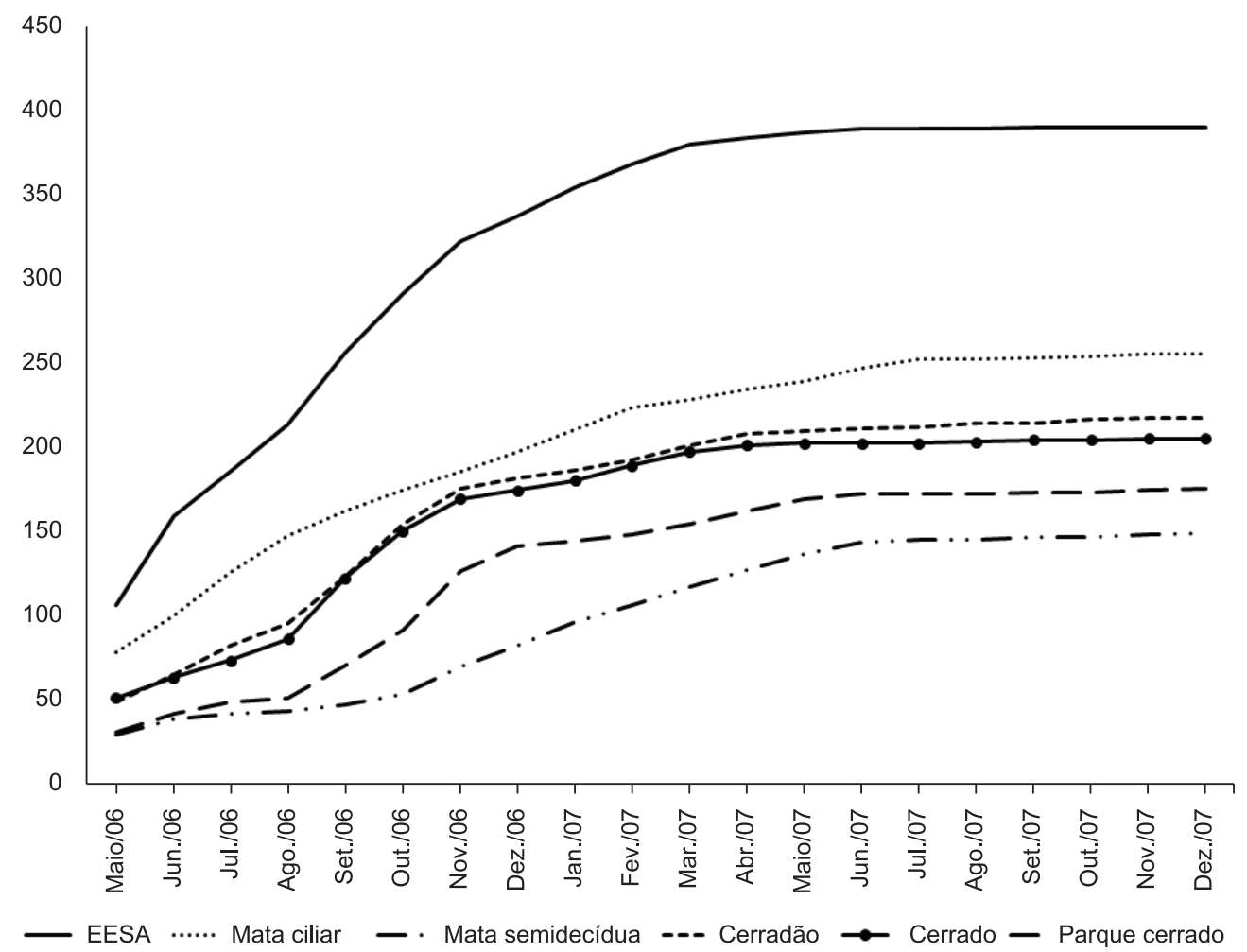

Figura 3. Curva do coletor observada que mostra o acúmulo de espécies registradas nas áreas amostradas de forma sistemática e da ESEC Serra das Araras, no eixo das abscissas temos os meses de amostragem e no das ordenadas temos o número acumulativo de espécies registrados durante o período de estudo.

Figure 3. Collector's Curve indicates an accumulation of species registered in the areas sampled in a systematic basis and the EESA, the x-axis indicates the month of sampling and in the ordinate shows the cumulative number of species recorded during the study period.

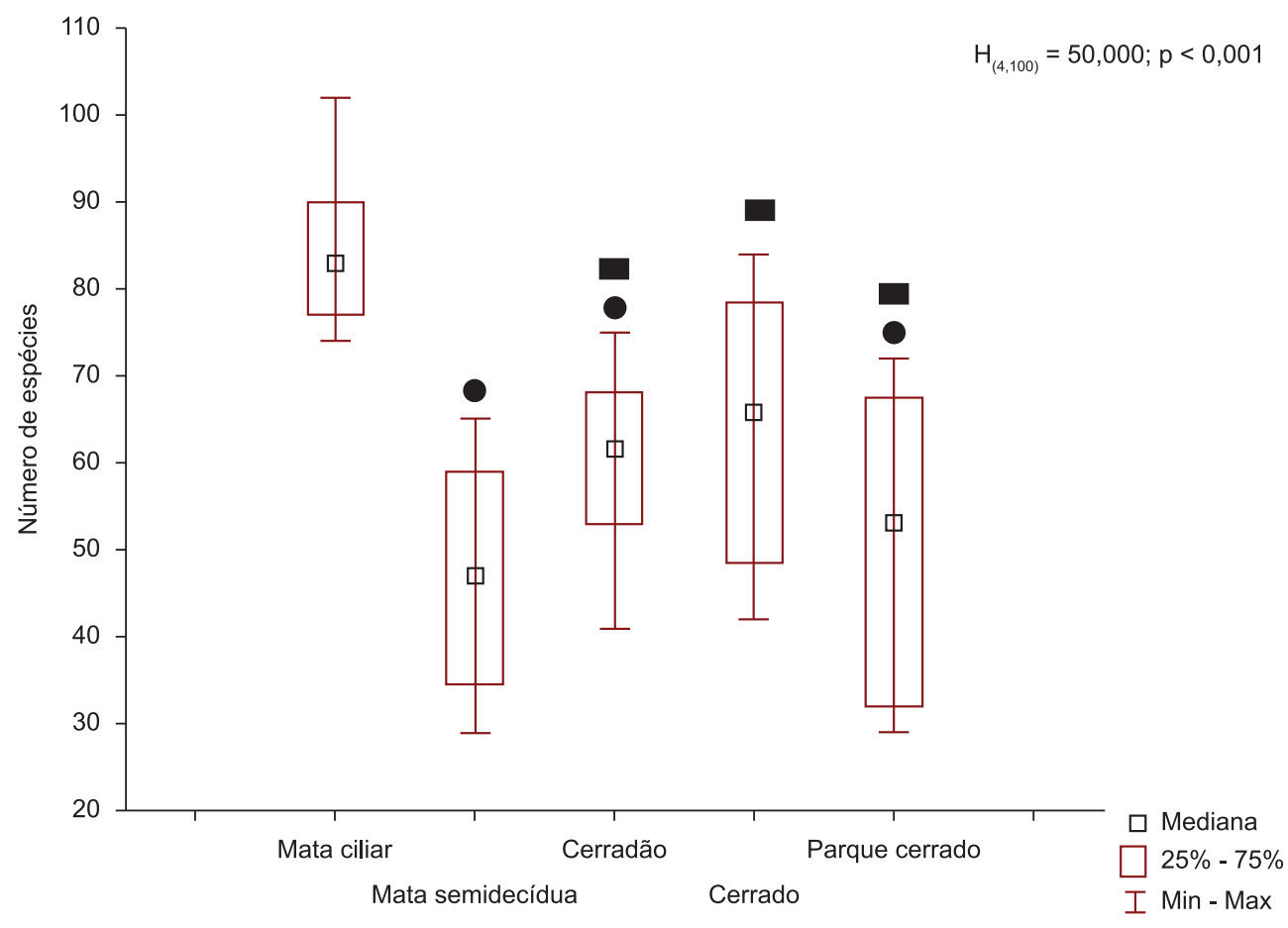

Fitofisionomia

Figura 4. Box-plot do número de espécies de aves observadas nas cinco fitofisionomias amostradas na Estação Ecológica Serra das Araras. Os símbolos indicam as áreas que não apresentaram diferenças das médias quando comparadas pelo teste de Tukey.

Figure 4. Box-plot of the number of bird species found in five vegetation types sampled in the Ecological Station of Serra das Araras. The symbols that indicate the areas that showed no differences of means compared by Tukey test. 


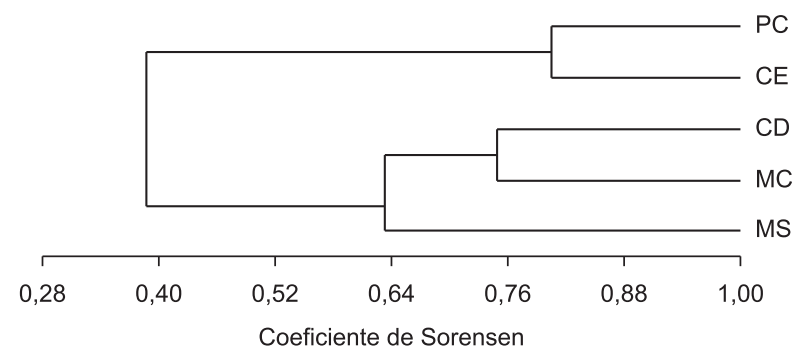

Figura 5. Dendrograma do índice de similaridade de Sörensen para as áreas amostradas de forma sistemática em que: MS (mata semidecídua), MC (mata Ciliar), CD (cerradão), CE (cerrado sentido restrito) e PC (parque cerrado).

Figure 5. Sorensen's similarity coefficient for the areas sampled in a systematic basis in which: MS (semi-deciduous forest), MC (riparian forest), CD (cerradão), CE (cerrado sentido restrito) and PC (parque cerrado).

O registro de 13 espécies de aves endêmicas do Cerrado, que representam 43,3\% das espécies endêmicas desse Bioma (Silva 1995, 1997, Cavalcanti 1999, Silva \& Santos 2005) e das sete espécies nacionalmente ameaçadas de extinção ressalta a importância da Estação Ecológica Serra das Araras na proteção dessas aves. Devido a sua particularidade, o registro de C. cyanopis merece destaque. Ele foi realizado no campo rupestre (Figura $2 \mathrm{~g}$ ) em março de 2007, por volta das oito horas, quando um único indivíduo foi avistado junto com três indivíduos de C. talpacoti que bebiam água numa poça em meio as rochas. No ano de 2011 fora montada uma equipe para tentar documentar a espécie durante duas viagens de sete dias, entretanto não foi obtido sucesso. Os registros realizados por Silva \& Oniki (1988) não indica o local, somente que fora nos limites da EESA. Willis \& Oniki (1990) esclarecem que os registros foram realizados em "campos cerrados". A elaboração de um plano de conservação para essa espécie deve ser considerado dentre os programas de conservação de espécies nacionalmente ameaçadas de extinção.

A presença de alguns elementos do Pantanal e a influência de elementos amazônicos, quando considerado Silva (1996), pode sugerir que a Província Serrana atue como um corredor entre o Cerrado e esses outros dois biomas. Dessa maneira, além dos extensos corredores ribeirinhos, conforme apontado por Silva \& Santos (2005); a preservação dessa cadeia de serras é outra estratégia para conservação dessas aves na região, mesmo que sua origem tenha sido relictual. A partir disso, ficam as sugestões de ampliação da ESEC Serra das Araras para sua região nordeste e sudoeste (vale do Rio Jauquara), a criação de um mosaico de unidades de conservação ao longo da Província Serrana e a sugestão de futuros estudos nessa cadeia de montanhas para corroborar ou negar essa "sugestão".

A partir dos resultados apresentados e discutidos, conclui-se que a Estação Ecológica Serra das Araras é uma das áreas no Cerrado com maior riqueza de espécies de aves registradas até o momento; e que além de preservar um elevado número, a presença de espécies endêmicas e nacionalmente ameaçadas de extinção faz dessa área protegida um local de grande importância para a conservação desse grupo animal, o que corrobora o proposto Oliveira et al. (2007) e Pinto et al. (2008), os quais indicam essa região como área prioritária para conservação de aves no Cerrado.

\section{Agradecimentos}

Ao colega Fabiano Ficagna de Oliveira pelo auxílio com a identificação de algumas espécies. Ao Nuno Rodrigues da Silva pelo auxílio na elaboração do mapa e a Carolina Pöter de Castro pelo auxílio com o abstract. Aos dois referis anônimos por suas críticas e sugestões para o melhoramento da qualidade do manuscrito.

\section{Referências Bibliográficas}

ALMEIDA, A., COUTO, H.T.Z. \& ALMEIDA, A.F. 2003. Diversidade beta de aves em hábitats secundários da Pré-Amazônia maranhense e interação com modelos nulos. Ararajuba 11(1):157-171.

ANTAS, P.T.Z. 1995. Aves do Parque Nacional de Brasília. IBAMA, Brasília.

ARGEL-DE-OLIVEIRA, M.M. 1993. Publicar ou não Publicar? Listas de espécies são necessárias? Bol. CEO 9:35-40.

AUGUST, P.V. 1983. The role of habitat complexity and heterogeneity in structuring tropical mammal communities. Ecology 64(6):1495-1507. http://dx.doi.org/10.2307/1937504

BAGNO, M.A. 1998. As aves da Estação Ecológica de Águas Emendadas. In Vertebrados da Estação Ecológica de Águas Emendadas - História Natural e Ecologia em um Fragmento de Cerrado do Brasil Central (J. Marinho-Filho, F. Rodrigues, M. Guimarães, eds.). SEMATEC, IEMA, IBAMA, Brasília, p.22-33.

BLAMIRES, D., VALGAS, A.B. \& BISPO, P.C. 2001. Estrutura da comunidade de aves da Fazenda Bonsucesso, município de Caldazinha, Goiás, Brasil. Tangara 1(3):101-113.

BRASIL. Ministério do Meio Ambiente. 1999. Termo de Compromisso visando a formulação e a implementação do Plano de Ação Integrado para os Biomas Cerrado e Pantanal. Ministério do Meio Ambiente, SECEX, Secretaria de Biodiversidade e Florestas, Brasilia.

CAVALCANTI, R.B. 1988. Conservation of birds in the Cerrado of Central Brazil. ICBP Technical Publication 7:59-66.

CAVALCANTI, R.B. 1992. The importance of Forest edges in the ecology of open contry Cerrado Birds. In (J.P. Furley \& J.A. Ratter, eds.). Chapman and Hall, London.

CAVALCANTI, R.B. (Coord.). 1999. Ações Prioritárias a Conservação da Biodiversidade do Cerrado e Pantanal. Ministério do Meio Ambiente, Brasília.

CAVARZERE, V., MORAES, G.P. \& DONATELLI, R.J. 2009. Avigauna da Estação Ecológica dos Caetetus, interior de São Paulo, Brasil. Pap. Avulsos Zool. 49(35):477-485.

COMITÊBRASILEIRO DE REGISTROS ORNITOLÓGICOS-CBRO. 2011 Listas das aves do Brasil. 10. ed. CBRO. Disponível em http://www.cbro. org.br (último acesso em 01/09/2011).

COUTINHO, L.M. 1978. O conceito de cerrado. Rev. Bras. Bot. 1:17-23.

CURCINO, A., SANT'ANA, C.E.R. \& HEMING, N.M. 2007. Comparação de três comunidades de aves na região de Niquelândia, GO. Rev. Bras. Ornit. 15(4):574-584.

EKEN, G., BENNUN, L., BROOKS, T.M., DARWALL, D., FISHPOOL, L.D.C., FOSTER, M., KNOX, D., LANGHAMMER, P., MATIKU, P., RADFORD, E., SALAMAN, P., SECHREST, W., SMITH, M.L., SPECTOR, S. \& TORDOFF, A. 2004. Key Biodiversity Areas as Site Conservation Targets. BioScience 54:1110-1118. http://dx.doi. org/10.1641/0006-3568(2004)054[1110:KBAASC]2.0.CO;2

FARIA, L.C.P., CARRARA, L.A. \& AMAARAL, F.Q. 2009. The birds of Fazenda Brejão: a conservation priority área of Cerrado in northwestern Minas Gerais, Brasil. Biota Neotrop. 9(3): http://www.biotaneotropica. org.br/v9ne/en/abstract?inventory+bn01109032009 (último acesso em 01/09/2011).

FERGUSSON-LEES, J., CHRISTIE, D.A., FRANKLIN, K., MEAD, D. \& BURTON, P. 2000. Raptors of the World. Helm Identification Guides. Christopher Helm, London.

FERREIRA, J.D., COSTA, L.M. \& RODRIGUES, M. 2009. Aves de um remanescente florestal do Quadrilátero Ferrífero, Minas Gerais. Biot. Neotrop. 9(3): http://www.biotaneotropica.org.br/von3/en/ abstract?article+bn00509032009 (último acesso em 01/09/2011).

INSTITUTO BRASILEIRO DO MEIO AMBIENTE E DOS RECURSOS NATURAIS RENOVÁVEIS - IBAMA. Lista de Espécies da Fauna Brasileira Ameaçadas de Extinção. Disponível em: http://www.mma.gov. br/port/sbf/fauna/lista.html (último acesso em 25/01/2005). 
KATTAN, G.H., ALVAREZ-LÓPEZ, H. \& GIRALDO, M. 1994. Forest fragmentation and bird extinction: San Antonio eiguty years later. Conserv. Biol. 8(1):138-146. http://dx.doi.org/10.1046/j.15231739.1994.08010138.x

LOPES, L.E. \& BRAZ, V.S. 2007. Aves da região de Pedro Afonso, Tocantins, Brasil. Rev. Bras. Ornit. 15(4):530-537.

LOPES, L.E., LEITE, L., PINHO, J.B. \& GOES, R. 2005. New bird records to the Estação Ecológica de Águas Emendadas, Planaltina, Distrito Federal. Ararajuba 13:107-108.

LOPES, L.E., PINHO, J.B., BERNARDO, B. OLIVEIRA, F.F., BERNARDO, J., FERREIRA, L.P., VASCONCELOS, M.F., COELHO, M.M., NOBREGA, P.F.A. \& RUBIO, T.C. 2009. Aves das Chapadas dos Guimarães, Mato Grosso, Brasil. Pap. Avulsos Zool. 49(2):9-47.

MacARTHUR, R.H., MacARTHUR, J.W. \& PREER, J. 1962. On bird species diversity: II predictions of bird census from habitat measurements. The Am. Nat. 96(8):167-174. http://dx.doi.org/10.1086/282219

MACHADO, R.B. \& LAMAS, I.R. 1996. Avifauna associada a um reflorestamento de eucalipto no município de Antônio Dias (MG). Ararajuba 4(1):15-22.

MARÇAL-JÚNIOR, O.M., FRANCHIN, A.G., ALTEFF, E.F., SILVAJÚNIOR, E.L. \& MELO, C. 2006. Levantamento da avifauna na Reserva Ecológica Panga (Uberlândia, MG, Brasil). Biosc. J. 25(6):149-164.

MARINI, M.A. 2001. Effects of forest fragmentation on birds of the Cerrado region, Brazil. Bird conserv. Int. 11:11-23. http://dx.doi.org/10.1017/ S0959270901001034

MELO-JÚNIOR, T.A., VASCONCELOS, M.F., FERNANDES, G.W. \& MARINI, M.Â. 2001. Birds species distribution and conservations in Serra do Cipó, Minas Gerais, Brazil. Bird conserv. Int. 11:189-204.

MOTA-JÚNIOR, J.C., GRANZINOLLI, M.A.M. \& DEVELEY, P.F. 2008. Aves da Estação Ecológica de Itirapina, estado de São Paulo, Brasil. Biota Neotrop. 8(3): http://www.biotaneotropica.org.br/v8n3/en/abstract?inve ntory+bn00308032008 (ultimo acesso em 01/09/2011).

MYERS, S., MITTERMEIER, R.A., FONSECA. G.A.B. \& KENT, J. 2000. Biodiversity hotspotos for conservation priorities. Nature 403:853-858. http://dx.doi.org/10.1038/35002501

NUNES, J.P. \& PACHECO, N. R. 2004. Informação geográfica e modelação para a gestão de bacias hidrográficas - consequências das alterações climáticas para os processos hidrológicos e erosivos: potenciar a informação geográfica para a gestão ambiental. In Anais do Encontro de Utilizadores de Informação Geográfica. Associação dos Utilizadores de Sistemas de Informação Geográfica, p.13.

OLIVEIRA, G., BARRETO, B., PINTO, M.P., DINIZ-FILHO, J.A.F. \& BLAMIRES, D. 2007. Padrões espaciais de diversidade da Família Emberezidae (Aves: Passeriformes) e seleção de áreas prioritárias para conservação no Cerrado. Lundiana 8(2):97-106.

PACHECO, J.F. \& OLMOS, F. 2006. As aves do Tocantins 1: região sudeste. Rev. Bras. Ornit. 14:55-71.

PARRINI, R., RAPOSO, M.A., PACHECO, J.F., CARVALHÃOES, A.M.P., MELO-JÚNIOR, T.A., FONSECA, P.S.M. \& MINNS, J. 1999. Birdos of the Chapada Diamantina, Bahia, Brazil. Cotinga 11:86-95.

PIANKA, E.R. 2000. Evolutionary ecology. 6th ed. Adison Wesley Educational Publishers, San Francisco.

PINHEIRO, R.T. \& DORNAS, T. 2009. Distribuição e conservação das aves da região do Cantão, Tocantins: ecótono Amazônia/Cerrado. Biot. Neotrop. 9 (1): http://www.biotaneotropica.org.br/v9n1/en/abstract?inv entory+bn02609012009 (último acesso em 01/09/2011).

PINTO, M.P., DINIZ-FILHO, J.A.F., BINI, L.M., BLAMIRES, D. \& RANGEL, T.F.L.V.B. 2008. Biodiversity surrogate groups and conservation priority áreas: birds of the Brazilian Cerrado. Diversity Distrib. 14:78-86. http://dx.doi.org/10.1111/j.1472-4642.2007.00421.x
PIVATTO, M.A.C., MANÇO, D.G., STRAUBE, F.C., URBEN-FILHO, A. \& MILANO, M. 2006. Aves do Planalto da Bodoquena, estado do Mato Grosso do Sul (Brasil). Atual. Ornitol. 129. http://www.ao.com.br (último acesso em 01/09/2011).

PURIFICAÇÃO, K.N. \& CASTILHO, L.S. 2009. Avifauna de quatro fitofisionomias de Cerrado do Parque Estadual da Serra Azul, Barra do Garças/MT. In Anais do IX Congresso de Ecologia do Brasil. São Lourenço, p.1-3.

REGO, M.A., SILVEIRA, L.F., PIACENTINI, V.Q., SCHUNC, F., MACHADO, É., PINHEIRO, R.T. \& REIS, E. 2011. As Aves na Estação Ecológica Serra Geral Tocantins, centro do Braisil. Biot. Neotrop. 2011 11(1): http://www.biotaneotropica.org.br/v11n1/en/ abstract?article+bn03711012011 (Último acesso em 01/09/2011)

RIBEIRO, J.F. \& WALTER, B.M.T. 2008. As Principais fitofisionomias do Bioma Cerrado. In Cerrado: ecologia e flora (S.M. Sano, S.P. Almeida \& J.F. Ribeiro, eds.). Embrapa Cerrados, Embrapa Informação Tecnológica, Brasília, p.151-213.

RIBON, R., ESTEVÃO, G.R.M., SIMON, J.E., SILVA, J.E., PACHECO, S. \& PINHEIRO, R.T. 1995. Aves do cerrado de Três Marias, estado de Minas Gerais. Ceres 42(242):344-352.

RIDGELY, R.S. \& TUDOR, G. 1989. The birds of South America: The oscine passerines. Austin, University of Texas Press, v.1.

RIDGELY, R.S. \& TUDOR, G. 1994. The birds of South America: The suboscine passerines. Austin, University of Texas Press, v.2.

RODRIGUES, M. CARRARA, L.A., FARIA, L.P. \& GOMES, H.B. 2005. Aves do Parque Nacional da Serra do Cipó: o Vale do Rio Cipó, Minas Gerais, Brasil. Rev. Bras. Zool. 22(2): 326-338. http://dx.doi.org/10.1590/ S0101-81752005000200005

RODRIGUES, M., FREITAS, G.H.S., COSTA, L.M., DIAS, D.F., VARELA, M.L.M. \& RODRIGUES, L.C. 2011. Avifauna, Alto Palácio, Serra do Cipó National Park, state of Minas Gerais, southeastern Brazil. Check List 7(2):151-161.

ROSS, J.L.S. 1991. O Contexto Geotectônico e a Morfogênese da Província Serrana de Mato Grosso. Ver. IG, São Paulo 12(1-2): 21-37.

SERVIÇO FLORESTAL BRASILEIRO - SBF. 2011. Florestas do Brasil em resumo 2010: dados de 2005-2010. SBF, Brasília, 152p.

SICK, H. 1966. As Aves do Cerrado como Fauna Arborícola. An. Acad. Bras. Cienc. 38(2):355-363.

SILVA, J.C.M. \& BATES, J.M. 2002. Biogeografic patterns and conservation in the South American Cerrado: a tropical Savanna hotspot. BioScience 52(3):225-233. http://dx.doi.org/10.1641/00063568(2002)052[0225:BPACIT]2.0.CO;2

SILVA, J.M.C. \& ONIKI, Y. 1988. Lista Preliminar da avifauna da Estação Ecológica Serra das Araras, Mato Grosso, Brasil. Bol. Mus. Para. Emilio Goeldi, Zool. 4(2):123-143.

SILVA, J.M.C. \& SANTOS, M.P.D. 2005. A importância relativa dos processos biogeográficos na formação da avifauna do Cerrado e de outros biomas brasileiros. In Cerrado: Ecologia, Biodiversidade e Conservação (A. Scariot, J.M.C. Silva \& J.M. Felfili, org.). Brasília, Ministério do Meio Ambiente, 439p., p.221-233.

SILVA, J.M.C. 1995. Birds of the Cerrado Region, South America. Steenstrupia 21:69-92.

SILVA, J.M.C. 1997. Endemic bird species and conservation in de Cerrado Region, South America. Biodivers. Conserv. 6:435-450. http://dx.doi. org/10.1023/A:1018368809116

SILVA, J.M.C. 1996. Distribuition of Amazonian and Atlantic birds in gallery forests of the Cerrado region, South America. Ornitol. Neotrop. 7(1):1-18.

SILVEIRA, L.F. \& D'HORTA, F.M. 2002. A Avifauna da região de Vila Bela da Santíssima Trindade, Mato Grosso. Pap. Avulsos Zool. 42(10):265-286.

SILVEIRA, L.F. 1998 The birds of Serra da Canastra National Parkand adjacent areas, Minas Gerais, Brazil. Cotinga 10:55-63.

SILVEIRA, L.F., BEISIEGEL, B.M., CURCIO, F.F., VALDUJO, P.H., DIXO, M., VERDADE, V.K., MATTOX, G.M.T. \& CUNNINGHAM, P.T.M. 2010. Para que servem os inventários de fauna? Est. Avançados 24(68):173-207. 
SOUZA, D.G.S. 1998. Todas as aves do Brasil - Guia de campo para identificação. Editora Dal, Bahia.

STOTZ, D.F., FITZPATRICK, J.W., PARKER III, T. \& MOSKOVITS, D.K. 1996. Neotropical birds: Ecology and Conservation. University of Chicago Press, Chicago.

R DEVELOPMENT CORE TEAM. 2011. R: A language and environment for statistical computing. R Foundation for Statistical Computing, Vienna, Austria. http://www.R-project.org/.

TUBELIS, D.P. \& CAVALCANTI, R.B. 2000. A comparison of bird communities in natural and disturbed non-wetland open habitats in the Cerrado's central region, Brazil. Bird conserve. Int. 10:331-350.
TUBELIS, D.P. \& CAVALCANTI, R.B. 2001. Community similarity and abundance of bird species in open habitats of a Central Brasilian Cerrado. Ornitol. Neotrop. 12:57-73.

VIELLIARD, J. 1995a. Guia Sonoro das aves do Brasil, CD 1. UNICAMP, MMS Estúdio, Campinas.

VIELLIARD, J. 1995b. Canto de aves do Brasil. UNICAMP, MMS Estúdio, Campinas.

VIELLIARD, J. 1999. Aves do Pantanal. UNICAMP, MMS Estúdio, Campinas.

WILLIS, E.O. \& ONIKI, Y. 1990. Levantamento preliminar das aves de inverno em dez áreas do sudoeste de Mato Grosso, Brasil. Ararajuba 1:19-38.

Recebido em 27/09/2011

Versão reformulada recebida em 10/08/2012

Publicado em 19/08/2011 